https://helda.helsinki.fi

\title{
Alphavirus polymerase and RNA replication
}

\section{Pietilä, Maija}

2017-04-15

Pietilä , M , Hellström , K \& Ahola , T 2017 , ' Alphavirus polymerase and RNA replication ' , Virus Research, vol. 234 , pp. 44-57 . https://doi.org/10.1016/j.virusres.2017.01.007

http://hdl.handle.net/10138/234230

https://doi.org/10.1016/j.virusres.2017.01.007

cc_by_nc_nd

acceptedVersion

Downloaded from Helda, University of Helsinki institutional repository.

This is an electronic reprint of the original article.

This reprint may differ from the original in pagination and typographic detail.

Please cite the original version. 


\title{
Alphavirus polymerase and RNA replication
}

\author{
Maija K. Pietilä*, Kirsi Hellström* and Tero Ahola\#
}

\author{
Department of Food and Environmental Sciences \\ University of Helsinki \\ Viikinkaari 9 (PO Box 56), 00014 Helsinki, Finland \\ \#corresponding author, e-mail: tero.ahola@helsinki.fi \\ *with equal contribution
}

Keywords: chikungunya virus; replication complex; membrane; antiviral; host factor; in vitro RNA synthesis

\begin{abstract}
Abbreviations (note that abbreviations for viruses are listed in Table 1): CPC, cyclopentenylcytosine; CPV, cytopathic vacuole; CSE, conserved sequence element; DI, defective interfering; dsRNA, doublestranded RNA; FXR Fragile X syndrome family proteins; G3BP, Ras-GTPase-activating protein (SH3 domain)-binding protein; hnRNP, heterogeneous nuclear ribonucleoprotein; ns, nonstructural; nsP, nonstructural protein; PABP, poly(A)-binding protein; PFZ, pyrazofurin; PI3K, phosphatidylinositol-3kinase; RC, replication complex; RdRp, RNA-dependent RNA polymerase; RF, replicative form; RI, replicative intermediate; SH3, Src-homology 3; sSRNA, single-stranded RNA; TATase, terminal adenylyltransferase; ts, temperature-sensitive; UTR, untranslated region
\end{abstract}

\section{Abstract}

Alphaviruses are typically arthropod-borne, and many are important pathogens such as chikungunya virus. Alphaviruses encode four nonstructural proteins (nsP1 to 4), initially produced as a polyprotein P1234. nsP4 is the core RNA-dependent RNA polymerase but all four nsPs are required for RNA synthesis. The early replication complex (RC) formed by the polyprotein P123 and nsP4 synthesizes minus RNA strands, and the late RC composed of fully processed nsP1 to nsP4 is responsible for the production of genomic and subgenomic plus strands. Different parts of nsP4 recognize the promoters for minus and plus strands but the binding also requires the other nsPs. The alphavirus polymerase has been purified and is capable of de novo RNA synthesis only in the presence of the other nsPs. The purified nsP4 also has terminal adenylyltransferase activity, which may generate the poly(A) tail at the $3^{\prime}$ end of the genome. Membrane association of the nsPs is vital for replication, and alphaviruses induce membrane invaginations called spherules, which form a microenvironment for RNA synthesis by concentrating replication components and protecting double-stranded RNA intermediates. The RCs isolated as crude membrane preparations are active in RNA synthesis 
in vitro, but high-resolution structure of the RC has not been achieved, and thus the arrangement of viral and possible host components remains unknown. For some alphaviruses, Ras-GTPase-activating protein (Src-homology 3 (SH3) domain)-binding proteins (G3BPs) and amphiphysins have been shown to be essential for RNA replication and are present in the RCs. Host factors offer an additional target for antivirals, as only few alphavirus polymerase inhibitors have been described.

\section{Alphavirus genome structure and replicase proteins}

Alphaviruses belong to the family Togaviridae together with the genus Rubivirus, which contains only one member, rubella virus (Table 1). Most alphaviruses are arthropod-borne viruses, and several are important human and/or animal pathogens, causing either fever, rash and arthritis (Old World alphaviruses, including chikungunya virus (CHIKV), Ross River virus (RRV) and Sindbis virus (SINV)), or encephalitis (New World alphaviruses, e.g. Venezuelan equine encephalitis virus (VEEV)). The alphavirus genome is a single positive-strand RNA of 11-12 kb with a $5^{\prime}$ cap0 structure and $3^{\prime}$ poly $(A)$. The two open reading frames encode the nonstructural (ns) or replicase polyprotein, and the structural polyprotein expressed via a subgenomic RNA (Fig. 1) (Strauss and Strauss, 1994). The ns polyprotein is processed in a highly regulated sequence to four final products, the nonstructural proteins (nsPs) 1-4 (Kääriäinen and Ahola, 2002). nsP1 is the viral capping enzyme and membrane anchor of the replication complex (RC) (Ahola and Kääriäinen, 1995; Spuul et al., 2007), while nsP2 is an RNA helicase and the protease responsible for the ns polyprotein processing (Das et al., 2014; Hardy and Strauss, 1989; Vasiljeva et al., 2003). nsP3 interacts with several host proteins and may modulate protein poly- and mono-ADP-ribosylation (Kim et al., 2016; Li et al., 2016), and nsP4 is the core viral RNA-dependent RNA polymerase (RdRp) (Rubach et al., 2009). Structural information is only available for the protease region of nsP2 and the folded $\mathrm{N}$-terminal portions of nsP3 (Shin et al., 2012).

The nsP4 polymerase is the most highly conserved protein in alphaviruses. Even nsP4s of the most diverged alphaviruses, the salmonid alphaviruses, are $\geq 50 \%$ identical in amino acid sequence with the other alphaviral nsP4s (Forrester et al., 2012; Weston et al., 2002). 
Within the mammalian/avian alphaviruses, the identities are even higher, e.g CHIKV nsP4 identity varying from 71\% (with Barmah Forest virus, BFV) to 91\% (with O'nyong-nyong virus, ONNV) (Khan et al., 2002). Alphavirus nsP4 is $~ 610$ amino acids in length, containing a large C-terminal domain related to other viral RdRps, and an alphavirus-specific $\sim 150$ amino acid $\mathrm{N}$-terminal domain. The $\mathrm{N}$-terminal domain is crucial for virus replication. It may be partially disordered structurally and it appears to interact with the other nsPs in the RCs (Rupp et al., 2011). Beyond the family Togaviridae, the alphaviruses belong to the large alphavirus-like superfamily that contains the animal hepeviruses and numerous genera of plant viruses (Koonin et al., 2015). All of these possess within their replicase proteins domains related to the capping enzyme nsP1, the helicase domain of nsP2 and the polymerase nsP4. When the predicted secondary structures of the RdRps from bromo- and tobamoviruses were compared to the partial crystal structure of poliovirus polymerase, these alphavirus superfamily members showed the typical RdRp structure with fingers, palm containing the GDD motif, and thumb domains (O'Reilly and Kao, 1998). In addition, comparison revealed a region preceding the fingers domain, which is unique to the RdRps and might be essential for the oligomerization of the polymerase. There are no structures available for the alphavirus nsP4, nor for any of the polymerases within the alphavirus-like superfamily. Now that structures have been solved for some of the negative-strand RNA virus polymerases (Pflug et al., 2017), the alphavirus superfamily may be the most significant branch of RdRps entirely lacking structural information. The biochemical characterization of nsP4 has also been challenging, as discussed in the next section. Therefore, much of this review will focus on the activities and properties of the alphavirus RC as a whole.

\section{Biochemical characteristics of nsP4: RNA synthesis and polyadenylation}

Most of the work on the replication and RNA synthesis of alphaviruses has been done with SINV and Semliki Forest virus (SFV) (Rupp et al., 2015). Together with the distant sequence relationship to other RdRps, analysis of temperature-sensitive (ts) mutants defective in RNA synthesis indicated that nsP4 is the catalytically active core polymerase subunit (Barton et al., 1988; Hahn et al., 1989a; Sawicki et al., 1990). 
SINV mutants ts 6 and ts 110 each have a single base substitution in nsP4 causing glycine

to glutamic acid change at position 153 or 324, respectively. These substitutions are located within highly conserved regions of nsP4 (Hahn et al., 1989a). In vitro RNA synthesis of ts6 shows that the RCs are stable at nonpermissive temperature but fail to elongate RNA strands indicating that the elongation capacity of the polymerase is inactivated (Barton et al., 1988). The in vitro RNA synthesis of the RCs is reactivated when they are returned to the permissive temperature.

SINV nsP4 has been expressed in E. coli and purified resulting in the full-length polymerase with an authentic N-terminal tyrosine and de novo RNA-synthesis activity but only when supplied with the polyprotein P123 (Rubach et al., 2009). Remarkably, the purified nsP4 is capable of forming the RCs with P123 resulting in the synthesis of discrete template-length minus strands from the provided plus-strand templates. Furthermore, nsP4 produced in bacteria has the same template requirements as the mammalian nsP4. The core catalytic domain ( $\Delta 97 \mathrm{nsP4}$, in which the $\mathrm{N}$-terminal 97 amino acids are deleted) has also been expressed in E. coli and purified as a monomer (Tomar et al., 2006). Interestingly, $\Delta 97 \mathrm{nsP4}$ lacks de novo copying activity, even when combined with the polyprotein P123 (Rubach et al., 2009; Tomar et al., 2006). Thus, the $97 \mathrm{~N}$-terminal residues seem to be crucial for the RdRp activity. The polyprotein P123 might be required in the template recognition or it may activate nsP4 through protein-protein interactions.

It is intriguing that nsP4 has also been shown to synthesize RNA in vitro in the absence of the other viral nsPs (Thal et al., 2007). SINV nsP4 was purified using detergent solubilisation of the membrane fraction from cells expressing uncleavable P123 and nsP4. The soluble form of nsP4 was able to synthesize template-length RNA from both minus- and plus-strand transcripts in the absence of the other nsPs. The authors suggest that the other components in the RC, determine the polarity of the RNA synthesized by nsP4. However, the ability of the detergent-solubilized nsP4 to synthesize RNA without the other nsPs is in conflict with the result that nsP4 purified from a bacterial expression system requires the polyprotein P123 for de novo RNA synthesis (Rubach et al., 2009). It is speculated that host proteins remaining in the same fraction as the soluble nsP4 may affect the RNA synthesis, but the bacterially produced nsP4 was unable to synthesize RNA even when combined with animal cell extracts (Rubach et al., 2009; Thal et al., 2007). 
Both the full-length and $\mathrm{N}$-terminally truncated nsP4 possess terminal, divalent cationdependent adenylyltransferase (TATase) activity, independent of P123, nsP1, nsP2, or nsP3 (Rubach et al., 2009; Tomar et al., 2006). At least 11-12 adenylate residues in the poly(A) tail next to the 3' conserved sequence element (CSE) are required for SINV RNA replication and efficient production of full-length minus strands (Hardy and Rice, 2005), and the TATase activity of nsP4 may play an important role in generating genome poly(A). However, it is not known if the poly(A) tail is added in a template-dependent or independent manner and if cellular and/or viral factors are responsible for the polyadenylation. Previously, it was suggested that the $5^{\prime}$ end of the minus strand is a poly $(U)$ tract and the initiation of the plusstrand synthesis occurs within this poly(U) tail resulting in the poly(A) tail (Frey and Strauss, 1978; Sawicki and Gomatos, 1976). However, more recent evidence shows that the initiation of the minus-strand synthesis occurs immediately after the poly(A) tail (Hardy, 2006) indicating that a template-independent mechanism is used to add the poly(A) tail. Furthermore, it has been observed that SINV poly $(A)$ tail is regenerated in vivo when RNA without the poly(A) tail is transfected into cells (Hill et al., 1997; Raju et al., 1999). A signal for this polyadenylation is located within the 29 3'-terminal nucleotides of the SINV genome (Raju et al., 1999).

Altogether, data imply that the TATase activity of nsP4 has a potential role in maintenance and repair of the poly(A) tail, and the work by Tomar et al. (2006) and Rubach et al. (2009) indicate that the poly(A) tail is added by a non-templated mechanism. The Nterminally truncated nsP4 ( $\triangle 97 \mathrm{nsP} 4)$ adds adenine to the 3 ' end of an acceptor RNA in the presence of different divalent cations, and mutations have confirmed the role of the alphavirus GDD motif in metal binding (Tomar et al., 2006). Other viral or host factors, or the $97 \mathrm{~N}$-terminal residues, might be needed for the template recognition as $\Delta 97 \mathrm{nsP} 4$ shows no sequence specificity in the TATase activity. Participation of other factors in nsP4 TATase activity is further supported by the observation that $\triangle 97 \mathrm{nsP} 4$ adds only two to four adenylate residues while the alphavirus poly $(\mathrm{A})$ tail is $\sim 70 \mathrm{nt}$. Furthermore, the full-length nsP4 shows more robust TATase activity than $\triangle 97 \mathrm{nsP4}$ demonstrating the importance of the $97 \mathrm{~N}$ terminal residues (Rubach et al., 2009). However, it remains to be studied whether nsP4 TATase activity is responsible for the in vivo polyadenylation of alphavirus RNA.

Mutations in nsP4 enable SINV to replicate in cells with lowered levels of rNTPs (Li et al., 2004; Lin et al., 2002; Lin et al., 2000). Pyrazofurin (PFZ), which is a cytidine analog 
decreasing UTP and CTP levels, inhibits SINV replication (Lin et al., 2000). Three amino acid substitutions in nsP4 (Met287 to Leu, Lys592 to lle, and Pro609 to Thr) are required for the PFZ-resistant phenotype of SINV. One of the substitutions (M287L) is located in the predicted fingers domain and the two others in the thumb domain of the polymerase, and it is suggested that these three substitutions, especially in the fingers, alter the rNTP-binding pocket increasing affinity for UTP and CTP. There is also a SINV mutant resistant to cyclopentenylcytosine (CPC), which reduces the level of CTP. One substitution in nsP4 (Leu585 to Phe) confers the resistance against CPC. This mutant has a lower $K_{m}$ for CTP compared to the wild type. The CPC-resistant mutant is sensitive to PFZ while the PFZ-resistant mutant is also resistant to CPC. As these two mutants have amino acid substitutions at different positions, it is suggested that the polymerase uses different amino acids to bind different nucleotides (Li et al., 2004).

High-fidelity variants of $\mathrm{nsP} 4$ as well as nsP2 are more resistant to inhibitors of nucleoside biosynthesis indicating that viruses could use fidelity changes to adapt to intracellular nucleotide depletions (Stapleford et al., 2015). Passaging of CHIKV in the presence of ribavirin, which is an RNA nucleoside analog, resulted in a mutant with a cysteineto-tyrosine substitution at position 483 of nsP4 (Coffey et al., 2011). This substitution of the conserved cysteine increased replication fidelity of the polymerase and thus decreased genetic diversity and fitness of the virus. The mutant is moderately attenuated in mammalian and mosquito hosts. A ribavirin- and 5-fluorouracil-resistant variant of CHIKV nsP2 has also been shown to increase the fidelity of the polymerase. This nsP2 variant exhibits delayed helicase activity, yet replication kinetics is increased (Stapleford et al., 2015). Thus, nsP2 and nsP4 may interact in the RCs to regulate the fidelity of the polymerase, and the interaction between these proteins has been observed in yeast-two hybrid and ELISA experiments (Sreejith et al., 2012; Stapleford et al., 2015).

Further substitutions at the Cys483 of CHIKV nsP4 have been generated to decrease the replication fidelity (Rozen-Gagnon et al., 2014). Polymerase variants with higher mutation frequencies were attenuated in vivo but showed no major replication defects in mammalian cells. On the other hand, these mutants had major defects in RNA synthesis in mosquito cells. The same effects were also observed with analogous mutations in SINV. Thus, manipulation of the polymerase fidelity offers a tool to attenuate alphaviruses in both mammalian and 
insect cells for example in order to develop vaccines (Coffey et al., 2011; Rozen-Gagnon et al., 2014).

The study of SINV RdRp mutators by Poirier et al. (2016) suggests a tight link between fidelity, recombination and defective interfering particle (DI) production. The mutators contain point mutations in viral polymerase and it was shown that the mutators have the ability to recombine in higher rate than wild-type viruses, leading to overproduction of DIs (Poirier et al., 2016). Recombination in alphaviruses was originally shown by the formation of DIs, virions with truncated viral genomes that accumulate during replication. They utilize fulllength viruses for multiplication and thus interfere with their replication (Poirier et al., 2016). The alphavirus DIs may contain duplicated, deleted or rearranged sequences and even cellular RNAs (Monroe and Schlesinger, 1983, 1984), but retain cis-acting sequence elements that are necessary for replication and packaging. Analyses of SFV and SINV DI genomes have provided important information on these critical elements needed for virus multiplication (reviewed in (Strauss and Strauss, 1994, 1997)).

Recombination seems to be somewhat less common in alphaviruses as compared to other groups of positive-strand RNA viruses, and both homologous as well as nonhomologous recombination has been described (Raju et al., 1995; Strauss and Strauss, 1997). In laboratory experiments, SINV constructs that were defective when introduced to the cells alone, were able to complement each other, giving rise to infectious recombinants (Weiss and Schlesinger, 1991). The recombination studies have given information especially on the $3^{\prime}$ end of the genome and its minimal requirements for minus strands synthesis (Hill et al., 1997) and on recombination hot spots (Hajjou et al., 1996).

Viruses must be able to repair their genomes and a mechanism for the 3 '-end repair pathway of alphaviruses has been suggested. Alphaviruses are able to add in vivo AU-rich sequences next to the poly(A) tail in genomes lacking the 3' CSE and infectious progeny viruses are made (Hill et al., 1997; Raju et al., 1999). The poly(A) tail is the prerequisite for these AU additions. It has been proposed that the polymerase stammers on AU-rich sequences and sliding, jumping, and stammering of the polymerase result in addition of such motifs to newly synthesized minus strands.

Alphavirus polymerases are considered to be viral template specific. SFV nsP4 is, however, at least to some extent, able to use other than viral RNA as a template to make double-stranded RNA (dsRNA) as it makes 5'-ppp dsRNA using host cell RNA as a template. 
(Nikonov et al., 2013). This, on the other hand, induces interferon-beta production mediated by retinoic acid-inducible gene I (RIG-I)-like receptors, and thus, this unspecific activity of the polymerase may be utilized by the host to restrict viral replication.

Alphavirus polymerase nsP4 has two major biochemical characteristics, de novo RNA synthesis and TATase activity. The former one requires the other nsPs and the latter one may generate the poly (A) tail typical of alphaviral genomes. The polymerase fidelity can be altered and mutations can also result in more frequent recombination. If a crystal structure of alphaviral polymerase is obtained, it would allow more robust comparison of this polymerase to other viral RdRps, beyond the sequence level. As the structures are more conserved than sequences (Mönttinen et al., 2014), new relationships between RdRp-encoding viruses could be formed.

\section{Minus-strand and plus-strand synthesizing replicases}

The core function of the viral polymerase lies in the genome replication but it is not the only element needed. The replication of alphaviruses is carefully orchestrated and requires all four nonstructural proteins that are processed from a polyprotein in a precise order during RNA synthesis (Fig. 2). The nonstructural proteins are translated as a polyprotein in the cytoplasm from the RNA genome released after virus entry. For most alphaviruses such as SINV, there is an opal (UGA) termination codon close to the C-terminus of nsP3 and thus two types of polyproteins, P123 and P1234, are produced. Of these only $10 \%$ is full-length polyprotein and P123 is produced in excess (Li and Rice, 1993). In contrast, SFV and ONNV have the stop codon replaced by arginine and only P1234 is produced (Strauss and Strauss, 1994; Takkinen, 1986). After translation, nsP4 is promptly cleaved by the protease activity residing in the Cterminus of nsP2 (de Groot et al., 1990). This cleavage is obligatory as the full-length form P1234 is not capable of any RNA synthesis (Kallio et al., 2016; Shirako and Strauss, 1994).

It has been demonstrated that nsP4 can also be provided separately by expressing it as an ubiquitin-fusion, which is rapidly cleaved to provide authentic nsP4 (Lemm et al., 1994; Spuul et al., 2011). The $\mathrm{N}$-terminal amino acid of nsP4 has a critical role in its functionality. In the wild type form, nsP4 contains tyrosine as the N-terminal amino acid (Strauss and Strauss, 1994) and excess nsP4 is rapidly degraded by the N-end rule pathway, a ubiquitin-dependent 
proteolysis (de Groot et al., 1991). In this process the protein's N-terminal amino acid plays a key role and tyrosine acts as a destabilizing residue (Varshavsky, 1992). Changing the $\mathrm{N}$ terminal amino acid to a nonaromatic residue in nsP4 is lethal for the virus, but an aromatic amino acid (Phe or Trp) or histidine rescues replication close to wild type level (Shirako and Strauss, 1998). An N-terminal Met results in viable virus with attenuated replication (Shirako and Strauss, 1998). Three second-site suppressor mutations resulting in amino acid substitutions allow the mutant SINV nsP4 with an N-terminal nonaromatic amino acid (Ala, Leu or Arg), to be functional. One suppressor mutation is located in nsP1 and two in nsP4 (Shirako et al., 2000). These results suggest that the role of the conserved tyrosine at the Nterminus of nsP4 is to interact with nsP1 and other nsP4 residues to allow proper folding and/or to interact with other viral or cellular factors to recognize the promoter and thus allow efficient initiation of the minus-strand synthesis. The ring structure of the aromatic amino acids may allow the right conformation for proper protein folding and protein-protein interactions (Shirako et al., 2000; Shirako and Strauss, 1998).

The early polymerase complex formed by $\mathrm{P} 123$ and nsP4 is dedicated to the synthesis of minus strand, using the viral genome as template (Lemm et al., 1994; Shirako and Strauss, 1994). This form of the complex is able to synthesize some plus-strand RNAs, but very inefficiently (Kallio et al., 2016; Lemm and Rice, 1993b). The following cleavage of nsP1 from P123 switches the complex in such a way that for a very short period, a complex of nsP1+P23+nsP4, seems to be capable of both minus and plus strand synthesis, but not subgenomic RNA synthesis (Jose et al., 2009; van der Heijden and Bol, 2002). The site between nsP2 and nsP3 is then cleaved in trans, leading to fully processed nonstructural proteins, forming the late RC. The switch to the late complex irreversibly "locks" the complex and it can only synthesize full length and subgenomic plus-strand RNAs from the minus strand. Thus in order to produce more minus strands, more polyprotein precursors should be translated. This has been demonstrated by treatment of cells with cyclohexamide which quickly shuts off the minus strand RNA synthesis (Sawicki and Sawicki, 1980; Sawicki and Sawicki, 1986). In infected mammalian cells, minus-strand synthesis occurs efficiently until 3 to 4 hours post infection (p.i.) and is then shut down as polyprotein cleavage becomes so rapid that new RCs can no longer be formed (Sawicki and Sawicki, 1980).

The polyprotein cleavage and its relation to strand-preference has been widely studied with the classic alphaviruses SFV and SINV. Different experimental strategies, such as 
cleavage-defective polyproteins and proteases (Lemm et al., 1998; Lemm and Rice, 1993b; Lemm et al., 1994; Shirako and Strauss, 1994; Vasiljeva et al., 2003), temperature-sensitive mutants (Hahn et al., 1989b; Sawicki et al., 1990; Sawicki and Sawicki, 1993; Sawicki et al., 1981; Wang et al., 1994) and reconstituted systems (Lemm and Rice, 1993a, b; Lemm et al., 1994; Spuul et al., 2011), have been used to gain a comprehensive set of results. It has been clearly shown that in order to synthetize minus-strands, the nonstructural proteins nsP1, nsP2 and nsP3 cannot be expressed separately but are needed as a polyprotein P123 (Lemm and Rice, 1993a; Lemm et al., 1994; Shirako and Strauss, 1994). In fact, even if P123 is expressed but is processed to individual proteins too fast, no RNA synthesis is detected (Lemm et al., 1994). A specific role for nsP1 in the minus-strand synthesis has been detected with SINV mutant ts11, which contains Ala-348 to Thr substitution in the nsP1 (Hahn et al., 1989b) and SFV mutants ts10 and ts14 (Lulla et al., 2008). Similarly, another mutant ts4 in SINV, mapping to nsP3 (Ala268 to Val), plays a role in minus-strand synthesis (Wang et al., 1994). All these mutants fail to synthesize minus-strand when infected cells are shifted from permissive to nonpermissive temperature.

Subsequent robust plus-strand RNA synthesis happens in the late RCs containing cleaved polyproteins, when subgenomic RNAs are produced in higher quantities than genomic RNAs (Keränen and Kääriäinen, 1979). Studies with uncleavable P123 or P23 have demonstrated that production of subgenomic RNA is impaired when fully processed nsPs are not present (Gorchakov et al., 2008a; Lemm and Rice, 1993b; Lemm et al., 1994; Shirako and Strauss, 1994). Interestingly, it has been possible to reactive minus-strand synthesis with a number of ts mutants in the absence of new protein synthesis (Sawicki and Sawicki, 1993; Suopanki et al., 1998), implying that the conformational changes is the nsPs between early and late RCs are so subtle that cleaved nsPs are able to switch back to the early complex form (reviewed in (Kääriäinen and Ahola, 2002)). A replicase containing uncleavable P123, with additional adaptive mutations, can also convert to the late form making only positive-strand RNAs (Gorchakov et al., 2008a).

The delicate interactions between the nsPs in the replication complex at the early and late stages are poorly understood due to the lack of structural data. A number of studies have addressed the role of nsP4 amino acid substitutions both in replication and in protein-protein interactions. It has been suggested that the conserved arginine 183 of SINV nsP4, which is predicted to be in the fingers domain, plays an important role in the initiation of the minus- 
strand synthesis and the interaction with host factors (Fata et al., 2002a). In addition, Gly83 to Leu substitution in the N-terminal domain of nsP4 also affects minus-strand RNA synthesis (Rupp et al., 2011). These minus-strand deficient mutants can be suppressed by second-site mutations in nsP1 indicating that nsP1 and nsP4 interact (Fata et al., 2002b; Rupp et al., 2011). Physical connection identified between nsP1 and nsP4 further confirms their interaction (Fata et al., 2002b; Lulla et al., 2008; Salonen et al., 2003; Shirako et al., 2000; Shirako and Strauss, 1998; Sreejith et al., 2012). Furthermore, second-site mutations in nsP2 and nsP3 suppress Asp41 to Ala substitution in nsP4, which results in defects in subgenomic RNA synthesis (Rupp et al., 2011). Altogether, the suppressor mutations imply that the $\mathrm{N}$-terminal domain of nsP4 interacts with all three other nsPs to activate RNA synthesis (Fata et al., 2002b; Rupp et al., 2011). It is suggested that this domain, due to its flexible nature, forms different contacts at different steps during the replication to allow the synthesis of different RNA species (Rupp et al., 2011).

A different type of experimental approach, relying on vaccinia virus based replication systems, has been very useful in understanding the different stages of RNA synthesis and the role of polyprotein cleavage (Lemm et al., 1994). More recently, a plasmid-based transreplication system was created for SFV (Spuul et al., 2011). The expression of polyprotein and template RNA takes place in T7 polymerase expressing cells from transfected DNAs. Thus the polyprotein or RNA expression is not dependent on replication, allowing studies with a range of modifications, including lethal ones (Kallio et al., 2016). It has been shown that capping activity, residing in nsP1, is not needed for negative strand synthesis, while many other enzymatic functions of the nsPs (helicase, protease and polymerase activities) and failure to bind to membranes totally abolished replication (Kallio et al., 2016). Similar systems have also been published for SINV (Frolova et al., 2010) and CHIKV (Utt et al., 2016). The latter has also been constructed as a cytomegalovirus-promoter driven system, allowing expression in wide range of cell types (Utt et al., 2016).

Several studies have shown that the positive-strand RNA template can be provided to the replicase in trans and is efficiently replicated (Lemm and Rice, 1993a, b; Spuul et al., 2011). However, attempts to similarly introduce minus-strand RNA templates have not succeeded (Hellström et al., 2016; Lemm et al., 1998), indicating that the replication can start only from plus-strand RNA. According to recent findings, minus strands are protected from RNase inside the spherules when RCs are isolated as membrane preparations (Kallio et al., 2016). This 
suggests that the minus strands are strictly segregated inside the spherules. Therefore, in normal situations, the minus strands would not be available as starting points for replication and there would be no advantage in having a mechanism to directly recruit the minus strand (Hellström et al., 2016).

\section{Promoter recognition by the polymerase}

A determining step of replication is when the polymerase recognizes and binds to the promoters. CSEs in the genome are thought to be recognized by the polymerase complex in the different stages of RNA replication (Rupp et al., 2015). The 5' end of the genome contains two CSEs, one in the $5^{\prime}$ untranslated region (UTR) and the other one within the nsP1 coding region, both forming stem-loop secondary structures (Frolov et al., 2001; Nickens and Hardy, 2008; Niesters and Strauss, 1990a, b; Ou et al., 1983). The two stem-loops formed by the 51nt CSE within the nsP1 region enhance both minus- and plus-strand RNA synthesis but they are dispensable for RNA replication. In contrast, the 5' UTR CSE contains essential promoter elements for both minus- and plus-strand synthesis. However, different alphaviruses might use different 5 ' elements for RNA synthesis as well as bind different host factors needed for RNA synthesis (Frolov et al., 2001; Gorchakov et al., 2004). The subgenomic promoter forms the third CSE, and a short CSE is located at the very $3^{\prime}$ end of the genome immediately preceding the poly $(\mathrm{A})$ tail. The core promoter of the minus-strand RNA synthesis is composed of the 3' CSE and poly(A) tail (Hardy and Rice, 2005; Rupp et al., 2015).

\subsection{Minus-strand RNA synthesis}

Efficient minus-strand RNA synthesis of SINV requires that 1 ) the length of the poly(A) tail is at least 11-12 residues, 2) the 3' CSE immediately precedes the poly(A) tail, and 3) the 3' 13 nucleotides of the 3' CSE are not changed (Hardy and Rice, 2005). It still remains unclear how alphaviruses initiate the minus-strand synthesis, but is has been suggested that they employ poly(A)-binding protein (PABP) and a similar genome-circularization mechanism as poliovirus, which uses a protein primer-dependent initiation on the 3' poly(A) tail (Frolov et al., 2001; Hardy and Rice, 2005; Peersen, 2017). It has been shown that the minimal length requirement of the SINV poly(A) tail is the same as that required for efficient binding of PABP (Deo et al., 1999; Hardy and Rice, 2005). Frolov et al. (2001) have suggested a model of how the 5' and 3' 
ends of the alphavirus genome interact to initiate minus-strand RNA synthesis. It is hypothesized that the viral RCs assemble on the $5^{\prime}$ end of the genome with translational machinery and possibly other host factors, and the interaction between the translation factors with PABP brings the 5' and 3' ends together. This is supported by competition assays where the 5' competitor RNA strongly inhibits minus-strand RNA synthesis, indicating that the 5' end binds limiting viral or cellular factors needed for the minus-strand RNA synthesis (Frolov et al., 2001).

Initiation site localization of the minus-strand RNA synthesis is dependent on the poly(A) tail and the last three 3' residues of the 3' CSE (Hardy, 2006). The predominant initiation site of the minus-strand synthesis is the cytidylate residue immediately preceding the poly $(A)$ tail, and this cytidylate is conserved among the alphaviruses (Adkins et al., 1998; Hardy, 2006). The following residues up to +12 from the initiating residue are hypothesized to be important for the transition from initiation to elongation and/or promoter recognition (Hardy, 2006).

\subsection{Plus-strand RNA synthesis}

The initiation site for the plus-strand RNA synthesis, both genomic and subgenomic, is highly conserved in the alphavirus genomes, and always starts with adenylate-uridylate, except that BFV has an extra uridylate residue at the $3^{\prime}$ end of the minus strand (Adkins et al., 1998). The promoter for the genomic RNA synthesis is located at the 3 ' end of the minus strand within the region corresponding to the 5' UTR stem loop sequence, and a complementary stem-loop structure has been predicted for the minus strand as well. The critical nucleotides for the SINV genomic promoter include those at positions 2 to 5 from the $3^{\prime}$ end of the minus strand (Frolov et al., 2001; Gorchakov et al., 2004; Li and Stollar, 2007; Niesters and Strauss, 1990a; Ou et al., 1983; Thal et al., 2007). The minimal promoter sequence required for the synthesis of SINV subgenomic RNA includes -19 to +5 nucleotides from the initiation site (Levis et al., 1990; Li et al., 2005), and nucleotides $-17,-14,-13$, and -11 relative to the initiation site are highly conserved among alphaviruses (Siegel et al., 1997).

Regulation of positive-strand RNAs synthesis, both genomic and subgenomic, is dependent on nsP4 as different sites of nsP4 bind the respective promoters ( $\mathrm{Li}$ and Stollar, 2004, 2007; Li et al., 2010). Crosslinking and gel mobility-shift assays revealed that the peptide corresponding to residues $329-334$ of nsP4 binds to the subgenomic promoter of SINV. This fragment is predicted to be on a $\beta$-strand in the fingers domain. Furthermore, nsP4 is able to 
bind to the subgenomic promoter only when all four nsPs are present suggesting that the other nsPs affect the conformation of nsP4 (Li and Stollar, 2004). The peptide corresponding to residues 531-538 of nsP4 binds the promoter for the genomic RNA synthesis (Li and Stollar, 2007; Li et al., 2010). Furthermore, mutations in nsP4, which abolish the subgenomic RNA synthesis, have no effect on the genomic RNA synthesis and vice versa (Li and Stollar, 2007; Li et al., 2010).

Alphaviruses typically make more subgenomic than genomic RNA in infected cells (Keränen and Kääriäinen, 1979). However, the PFZ- and CPC-resistant mutants of SINV synthesize more genomic than subgenomic RNA, and except for one, the mutations are in the region of both nsP4-coding sequence and subgenomic promoter (Li et al., 2008; Li et al., 2004; Li et al., 2010; Lin et al., 2002; Lin et al., 2000). It has been shown that the changed RNA ratio is mainly due to the changes in the promoter sequence (Li et al., 2010). The genomic / subgenomic RNA ratio made by these mutants in vitro is also affected by the nucleotide concentrations used in the reaction mixture indicating that the NTP concentrations affect which promoter the polymerase chooses. Thus, the cytoplasmic NTP concentration may affect the activity of the viral polymerase (Li et al., 2008; Li et al., 2010). Additionally, defects in subgenomic RNA synthesis are detected with some ts mutants like SFV ts4 and SINV ts15, ts17, ts18, ts24 and ts133 (Kääriäinen and Ahola, 2002; Sawicki and Sawicki, 1985; Suopanki et al., 1998) which all map to nsP2. Therefore, nsP2 may be required to recognize the subgenomic promoter in the minus strand RNA.

The requirement for an aromatic amino acid at the $\mathrm{N}$-terminus of $\mathrm{nsP} 4$ is also interesting with respect to the plus-strand RNA synthesis. Besides suppressor mutations in nsP1 or nsP4, addition of $A U, A \cup A$, or $A U U$ to the $5^{\prime}$ terminus of the genome or substitution of the third nucleotide of the genome, A for U, restore the activity of the SINV polymerase mutant with an N-terminal nonaromatic amino acid (Ala, Leu, or Met) (Shirako et al., 2003). It is speculated the polymerase mutants with a nonaromatic amino acid at the $\mathrm{N}$-terminus are unable to open the stem-loop structure at the 3 ' terminus of the minus strand to initiate plus-strand RNA synthesis, and the addition of AU-rich sequences or the $\mathrm{A}-\mathrm{U}$ substitution enables the polymerase mutants to work. Thus, it is proposed that the $\mathrm{N}$-terminus of the polymerase forms direct contacts with the 3 ' terminus of the minus strand to initiate plus-strand RNA synthesis (Shirako et al., 2003). However, minus-strand synthesis of the polymerase mutant 
must also be restored, and consequently the addition of these AU-rich sequences to the $5^{\prime}$ terminus of the genome most likely play a role also in minus-strand synthesis.

To summarize, the initiation sites of RNA synthesis are highly conserved in the alphavirus genomes. The 3' CSE and poly(A) tail form the core promoter of the minus-strand RNA, and the minus strand region corresponding to the 5' UTR stem loop forms the promoter for the genomic RNA. The CSE within the 5' UTR is important also for minus-strand RNA synthesis. Different sites of nsP4 bind the genomic and subgenomic promoters, and nsP2 may also play a role in subgenomic promoter recognition.

\section{Membrane association of replication - spherules}

Early studies with SFV by Grimley et al. already suggested that the replication of alphaviruses takes place in membrane-associated structures called spherules, located either on the plasma membrane or on the limiting membranes of type I cytopathic vacuoles (CPV-I), which are 6002000 nanometres in diameter (Grimley et al., 1968; Grimley et al., 1972). Spherules themselves are about 50-60 nm membrane invaginations that are connected to the cytoplasm by a narrow neck, allowing export of the nascent RNA (Fig. 3). The spherules contain dense material, presumably representing RNA (Froshauer et al., 1988). The association of nsPs (Froshauer et al., 1988; Kujala et al., 2001) and localization of dsRNA staining with spherules (Frolova et al., 2010; Spuul et al., 2010) have been nicely demonstrated, strengthening the view that they are the site of viral replication. The composition of the spherules is, however, poorly understood, and the amount of the nsPs in each spherule is unknown but has been estimated to be quite low (Frolova et al., 2010).

In addition to spherules, nsPs are found in different, but specific places, within the cells. Characteristic localization of nsP1 on the inner surface of the plasma membrane (Spuul et al., 2007) results from its palmitoylation (Laakkonen et al., 1996; Peränen et al., 1995) and membrane binding amphipathic peptide (Ahola et al., 2000; Ahola et al., 1999; Lampio et al., 2000). By contrast, nsP2 is found in the nucleus due to the nuclear localization signal in its Cterminus (Peränen et al., 1990; Rikkonen et al., 1992). The C-terminal motif of nsP3 on the other hand is responsible for binding cellular proteins such as Ras-GTPase-activating protein (SH3 domain)-binding proteins 1 and 2 (G3BP1 and G3BP2) (Fros et al., 2012; Panas et al., 
2012; Scholte et al., 2015) for Old World alphaviruses and Fragile $X$ syndrome family (FXR) proteins for New World alphaviruses like VEEV (Foy et al., 2013; Kim et al., 2016), leading to

478 formation of cytoplasmic aggregates where nsP3 accumulates (Frolova et al., 2006; 479 Gorchakov et al., 2008b). nsP4 is detected diffusely in the cytoplasm (Kujala et al., 2001), though most of it is quickly degraded as mentioned in above. However, a resistant, stable fraction of nsP4 has been detected and is postulated to be the one that is associated with RCs and is thus protected from degradation (de Groot et al., 1991). SINV nsP4 synthesized early in infection is more stable than the one made late in infection. This would be consistent with the hypothesis that assembly of nsP4 in the RCs protects the protein (de Groot et al., 1990; de Groot et al., 1991; Hardy et al., 1990; Hardy and Strauss, 1988). When lysates from SFVinfected cells are separated into a nuclear pellet and post-nuclear supernatant and the supernatant is further separated into a membrane and cytosolic fractions, most of SFV nsP4 is found in the membrane fraction (Peränen et al., 1988), indicating that the stable fraction of nsP4 is associated with membranes.

Spherules are part of the cellular membrane trafficking network. For example, SFV spherules are originally formed at the plasma membrane and are then internalized (Spuul et al., 2010), leading to spherule-lined, modified late endosomal/lysosomal CPVs that are often in the vicinity of rough endoplasmic reticulum (Froshauer et al., 1988). In a recent study, it was observed that in contrast to SFV, only a fraction of SINV spherules are internalized. However, some CPVs can be detected later during the SINV infection (Frolova et al., 2010). The internalization has been extensively studied with SFV and has been shown to be dependent on the activity of phosphatidylinositol-3-kinase (PI3K) (Spuul et al., 2010). In a recent study by Thaa et al. (2015) the RC internalization was compared between SFV and CHIKV and was further linked to the activation of PI3K-Akt-mTOR pathway. The study showed that the hyperphosphorylated/acidic domain of nsP3 is responsible for the pathway activation and that SFV and CHIKV greatly differed in this respect; while SFV boosted the pathway and showed efficient spherule internalization, CHIKV RCs stayed mostly at the plasma membrane. By exchanging the responsible domain from SFV to CHIKV, it was possible to obtain pathway boosting and RC internalization also with CHIKV (Thaa et al., 2015). In addition, the early steps during SFV spherule internalization need an intact actin network, while microtubules are necessary for later CPV accumulation in the perinuclear region. However, it has been shown that the internalization from the plasma membrane is not necessary for active replication, but 
is rather speculated to be involved in protecting virus production from cellular defence mechanisms (Spuul et al., 2010).

How the spherules are formed is still under study. For example, the exact mechanism and place of the RNA recruitment is not known but the RC needs to be membrane associated (Salonen et al., 2003; Spuul et al., 2007). It has been shown that all four replicase proteins need to be present for spherules to form. Active replication, or at least minus-strand synthesis is a prerequisite for spherule formation (Frolova et al., 2010; Hellström et al., 2016; Kallio et al., 2016; Spuul et al., 2011), in accordance with the studies where the inactivation of the polymerase nsP4 abolishes spherules (Kallio et al., 2016; Spuul et al., 2011; Utt et al., 2016). In a recent study, it was demonstrated that the spherule size could be manipulated by changing the template length (Kallio et al., 2013), thus indicating that the spherule is not a rigid structure but can be somewhat flexible. The role of host proteins in the spherule formation is largely unknown, but amphiphysins have been shown to participate in replication through nsP3 interactions. Since they are membrane binding proteins inducing positive curvature, it is possible that they participate in spherule formation where positive curvature is found on the neck structure (Neuvonen et al., 2011).

While the spherules are shown to locate on plasma membrane and CPVs for alphaviruses, the other positive-strand RNA viruses use other membrane types for their replication. Rubella virus, the only member of the genus Rubivirus is replicating on the lysososomal-originated CPVs as do alphaviruses, but not on the plasma membrane (Kujala et al., 1999). Brome mosaic virus, belonging to the alphavirus-like superfamily, induces spherules at the endoplasmic reticulum, which is also the site of invaginated vesicles formed by flaviviruses, e.g. dengue and West Nile virus. Instead, the spherules of the nodavirus Flock House virus are found on the outer mitochondrial membrane. Some plant viruses employ plant-specific membranes such as chloroplasts (turnip yellow mosaic virus) and peroxisomes (tomato bushy stunt virus) for the membranous RC formation (reviewed in (Paul and Bartenschlager, 2013; Romero-Brey and Bartenschlager, 2014; Stapleford and Miller, 2010)). Thus it is likely that even though the RC structures of these viruses resemble each other, the required cellular components significantly differ due to the variable intracellular location.

In spite of the development of advanced electron microscopy techniques and the extensive studies of many plus-strand viruses, the ultrastructure, molecular components or formation process of RCs are still largely unknown and many details are missing. The role of 
host proteins, cellular lipids and viral proteins and RNA in cellular membrane rearrangements for replication purposes are studied by several groups but clearly much is still to be found

542 (reviewed in (Miller and Krijnse-Locker, 2008; Reid et al., 2015a; Stapleford and Miller, 2010). In addition, the critical information of nonstructural protein interactions and their conformational changes within RCs during replication are mainly uncharacterized (Rupp et al., 2015). However, the reasons for membrane-associated replication seem to be generally accepted to be the concentration of necessary components to a restricted, specific cytoplasmic location, to provide scaffold of RC anchorage, to protect the viral RNA from cellular defence mechanisms and in some cases to link replication and subsequent virus assembly (Lorizate and Krausslich, 2011; Miller and Krijnse-Locker, 2008; Neufeldt et al., 2016; Salonen et al., 2005).

\section{Purification of replication complexes and in vitro RNA synthesis}

One of the outstanding questions in the alphavirus field is the structure and organization of the RCs as no high-resolution structure is available for the polymerase or the RC. As nsP4 recognizes and binds to the promoter sequences and is active in de novo RNA synthesis only in the presence of the other nsPs (Li and Stollar, 2004; Rubach et al., 2009), it is necessary to determine the structure of the active polymerase in association with the other nsPs, i.e. to determine the RC structure. Although no structure is yet available, several attempts have been made to purify the active RCs, and the polymerase functions have been studied using in vitro assays (Albulescu et al., 2014; Barton et al., 1991; Clewley and Kennedy, 1976; Gomatos et al., 1980; Lemm et al., 1998).

Most of the in vitro RNA-synthesizing activity is found in the membrane fraction prepared from infected cells. All four nsPs are also found in this fraction as well as most of the minus-strand RNA made in vivo as expected based on its most likely location in the membranous RCs (Albulescu et al., 2014; Barton et al., 1991; Clewley and Kennedy, 1976; Gomatos et al., 1980; Peränen et al., 1990; Peränen et al., 1988). Immunoprecipitation assays with SINV RCs showed that each anti-nsP antibody is able to pull down all four nsPs indicating that they form a tight complex (Barton et al., 1991). Most of the positive-strand RNA in cells is released from the RCs during the replication as it is mainly found in the cytosolic fraction 570 (Albulescu et al., 2014). 
The activity of the RCs in crude membrane fractions also indicates that all the host factors required for the RNA synthesis are present in the membrane fraction and no soluble

573 factors are needed. The in vitro RNA synthesis of CHIKV, SINV, and SFV results in the same RNA species as made in vivo; genomic and subgenomic single-stranded RNA (ssRNA) as well as dsRNA, which is in replicative form (RF) and/or replicative intermediates (RI) (Fig. 4) (Albulescu et al., 2014; Clewley and Kennedy, 1976; Michel and Gomatos, 1973; Sreevalsan and Yin, 1969). The majority of the in vitro synthesized RNA is in a single-stranded form, and CHIKV virus synthesizes three ssRNA species, all of positive-polarity: subgenomic and genomic RNA as well as RNA II which is a $\sim 7.5-\mathrm{kb}$ fragment preceding the subgenomic promoter (Albulescu et al., 2014; Clewley and Kennedy, 1976). Kinetics studies with SFV have shown that RF and RI dsRNA are synthesized first and serve as precursors for $26 \mathrm{~S}$ and $42 \mathrm{~S}$ ssRNAs, which are then synthesized at a linear rate (Michel and Gomatos, 1973). Both $26 \mathrm{~S}$ and $42 \mathrm{~S}$ ssRNAs synthesized in vitro are also methylated. The methylation occurs at the 5 ' terminus of the RNA strands and the cap structure is the same as in cells. Furthermore, the methylation activity co-purifies with the polymerase activity although the in vitro synthesis of ssRNAs is not dependent on the methylation (Cross and Gomatos, 1981).

The in vitro polymerase activity of CHIKV is relatively stable between 20 and $37^{\circ} \mathrm{C}$ reflecting the need of alphaviruses to replicate both in mosquito and mammalian cells. One third of the in vitro ssRNA made by CHIKV is in RIs and the rest is released from the RCs. The newly made RNA is rather stable indicating some mode of protection, and it is suggested that membrane association, polysomes, RNA structure, or its encapsidation provide this (Albulescu et al., 2014). For SFV, it has also been indicated that the RNA species synthesized in vitro are associated with some structures and do not occur in a free form (Michel and Gomatos, 1973).

Ultracentrifugation in sucrose-density gradients has been the main method to purify the alphavirus RCs. A cytoplasmic complex isolated from SINV-infected cells synthesized in vitro ssRNA, RF, and maybe also RIs, and membrane association of the structure was proposed based on the detergent and nuclease treatments (Sreevalsan and Yin, 1969). A similar approach was used to show that SFV polymerase activity, viral RNA, and CPVs enrich in the same fraction. Furthermore, as the isolated CPVs contained viral RNA, their central role in RNA replication was indicated (Friedman et al., 1972). In another SFV study, in vivo pulselabeled RNA and polymerase activity enriched in the smooth-membrane fraction, which 
synthesized and released $26 \mathrm{~S}$ and $42 \mathrm{~S}$ ssRNA. Addition of the cytosolic fraction to the smoothmembrane fraction did not increase the polymerase activity indicating that no soluble factors are needed for the in vitro RNA synthesis (Gomatos et al., 1980).

Detergent solubilisation has also been used in many approaches to purify the alphavirus RCs (Barton et al., 1991; Clewley and Kennedy, 1976; Gomatos et al., 1980; Ranki and Kääriäinen, 1979). However, all these protocols seem to affect the activity of the polymerase demonstrating the central role of membrane association. First, Clewley and Kennedy (1976) developed a multi-step purification scheme to purify SFV RCs by ultracentrifugation in density gradients, detergent solubilisation, and affinity chromatography. Although the polymerase was purified over 300 -fold, the recovery of activity was only about $5 \%$. Furthermore, purification products incorporated label only into RI or RF indicating that the release of ssRNA was affected and the polymerase was unable to initiate new strands during the in vitro synthesis (Clewley and Kennedy, 1976). The same inhibition of ssRNA release was observed when SFV RCs were solubilized from the smooth-membrane fraction, and the polymerase activity was reduced (Gomatos et al., 1980). Besides, only some of the nsPs were detected in the solubilized RCs (Clewley and Kennedy, 1976; Gomatos et al., 1980; Ranki and Kääriäinen, 1979). Detergent-solubilized RCs of SINV purified by ultracentrifugation in glycerol and sucrose gradients have been shown to contain all four nsPs but also these RCs incorporated label only into RIs (Barton et al., 1991).

It is clear that new approaches are needed to obtain highly purified RCs, which remain active in RNA synthesis. One such approach could be in vitro assembly as alphavirus nsPs are able to assemble into functional RCs in vitro, and the presence of an endogeonous template is not a prerequisite for the in vitro RNA synthesis. The membrane fraction containing the uncleavable polyprotein $\mathrm{P} 123$ and polymerase nsP4 of SINV is able to initiate and synthesize the minus-strand RNA when provided with an exogenous plus-strand RNA template (Lemm et al., 1998). Expression of nsP1 + uncleavable P23 + nsP4 also enables the in vitro minusstrand synthesis while cleavable P123 + nsP4 results in decreased RNA synthesis confirming that the polyprotein P23 is necessary for the minus-strand initiation complex. If soluble nsP4 is combined with the membrane fraction containing P123 and an exogenous template is added, in vitro RNA synthesis is also observed. In addition, nsP4 expressed and purified from E. coli is able to synthesize RNA in vitro when combined with the P123-membrane fraction 
interesting starting point to solve the structure. Activity-preserving purification method would then provide a tool to resolve the components, organization, and functional details of alphavirus RCs. Especially, such an approach would be invaluable in order to determine host factors crucial for the formation of functional RCs.

\section{Host factors and alphavirus polymerase complex}

Identities and exact roles of host proteins in alphavirus RNA synthesis remains an open question. Host proteins co-purify with the RCs (Barton et al., 1991; Clewley and Kennedy, 1976), and several host factors interact with alphavirus RNA or nsP-containing complexes (Fig. 5). Mass spectrometry has revealed that SINV nsP2-, nsP3-, or nsP4-containing complexes are rich in cytoskeleton proteins, chaperons, elongation factors, heterogeneous nuclear ribonucleoproteins (hnRNPs), tyrosine 3-monooxygenase/tryptophan 5-monooxygenase activation proteins (14-3-3 proteins), and ribosomal proteins (Atasheva et al., 2007; Cristea et al., 2006; Cristea et al., 2010; Frolova et al., 2006; Gorchakov et al., 2008b). A similar set of proteins, including RNA-binding (e.g. hnRNPs and G3BPs), cytoskeleton (e.g. tubulins), translation (e.g. eukaryotic translation elongation factors), folding (e.g. heat shock proteins), and 14-3-3 proteins, were enriched with CPVs isolated from SFV-infected cells (Ahlquist, 2006). The interaction with the host proteins is time-dependent suggesting that different host proteins are required for the multiple functions of nsP4 at different phases of infection such as the minus-strand synthesis early, and plus-strand synthesis later in infection (Cristea et al., 2006; Cristea et al., 2010). However, some of the identified host proteins may interact with nsPs which are not located in the RCs and thus do not affect the polymerase activity.

The number of the identified host factors in the SINV nsP3-containing complexes after a strong detergent treatment was considerably lower than in the other studies (Gorchakov et al., 2008b). G3BP1 and G3BP2, nucleic acid-binding protein YBX1, and heat shock protein HSC70 were detected in these samples (Fig. 5). The same nucleic acid-binding and heat shock proteins have also been detected in SINV nsP2-containing complexes (Atasheva et al., 2007). In addition, the heat shock protein HSP-90 has been identified in CHIKV nsP3- or nsP4containing complexes as well as in SINV nsP4-containing complexes (Fig. 5) (Cristea et al., 
2010; Rathore et al., 2014). Furthermore, silencing of HSP-90 inhibits CHIKV replication (Rathore et al., 2014).

In addition to the SINV nsP3-containig complexes, G3BPs have been detected in the SINV nsP2- and nsP4-containing complexes as well as in the SFV and CHIKV nsP3-containing complexes (Fig. 5) (Atasheva et al., 2007; Cristea et al., 2006; Cristea et al., 2010; Frolova et al., 2006; Gorchakov et al., 2008b; Kim et al., 2016; Panas et al., 2014; Panas et al., 2012). The role of G3BPs in SINV infection has been studied by their knockdown resulting in enhanced expression of the polyprotein but with minimal effects on RNA synthesis (Cristea et al., 2010). On the contrary, is has been shown for CHIKV that the depletion of G3BPs results in significantly reduced levels of the minus-strand RNA, and consequently the plus-strand RNA, and in reduced replication (Scholte et al., 2015). It was suggested that early in CHIKV infection G3BPs are essential in the switch from translation to genome replication by possibly removing the ribosomes from the viral RNA. The authors also show that late in CHIKV infection G3BPs are found in nsP3 aggregates which are proposed to prevent the formation of typical stress granules. Interestingly, the same authors also observed reduction in SINV replication in G3BPdepleted cells. It was speculated that G3BP2 levels may not have been low enough in previous studies to observe the inhibition of replication as it was shown that the knockdown of G3BP2 alone is enough to significantly reduce replication of both CHIKV and SINV. A recent study by Kim et al. demonstrated how New World and Old World alphaviruses differ in their host protein selection even though in both groups the stress-granule related proteins are used for RNA replication and RC assembly. While SINV and CHIKV replication were clearly reduced when both G3BP homologs were knocked out, similar effect was not seen with VEEV. Instead, the knockout of FXR family members was specifically affecting VEEV (Kim et al., 2016).

The complex formed by nsP3 and G3BP has shown to recruit sphingosine kinase 2 (SK2) (Fig. 5) (Reid et al., 2015b). In addition, SK2 co-localizes with dsRNA in the infected cells indicating its presence in the RCs, and most likely nsP3 is responsible for its recruitment to the RCs. Knockdown of SK2 inhibits CHIKV replication, and during infection SK2 has been shown to interact with proteins involved in mRNA processing and gene expression such as poly(C)-binding proteins (PCBPs) 1 and 2. The carboxy-terminal proline-rich motif of SFV, SINV, and CHIKV nsP3 binds SH3-domains of amphiphysin-1 and -2 (Fig. 5) (Neuvonen et al., 2011). The interaction is important for viral RNA replication, and as amphiphysins work in 
membrane dynamics (Graham and Kozlov, 2010), they might be important for spherule formation in alphavirus-infected cells (Neuvonen et al., 2011).

Several hnRNPs have been shown to relocalize during alphavirus infections and to interact with viral components (Ahlquist, 2002, 2006; Ahlquist et al., 2003; Balistreri et al., 2007; Cristea et al., 2006; Frolova et al., 2006; Gui et al., 2010). SINV nsP1, nsP2, and nsP3 as well as subgenomic RNA but not the genomic RNA co-immunoprecipitate with hnRNP K (Fig. 5), and based on the knockdown of hnRNP $\mathrm{K}$ it was speculated that hnRNP $\mathrm{K}$ facilitates transcription of the subgenomic RNA (Ahlquist et al., 2003). Knockdown of hnRNP A1 also results in reduced SINV replication (Balistreri et al., 2007). It was suggested that hnRNP A1 plays a role in SINV minus-strand RNA synthesis and viral RNA translation as it interacts with the 5' UTR. Furthermore, hnRNP A1 interacts with both SINV genomic and subgenomic promoters, and it has been shown that hnRNP A1 is required for the plus-strand, both genomic and subgenomic, RNA synthesis (Gui et al., 2010). Several hnRNPs were also recognized in the CPVs isolated from SFV-infected cells (Ahlquist, 2006). Silencing of RNAbinding proteins hnRNP M and C facilitated SFV, CHIKV, and SINV replication, while silencing of hnRNP K increased SFV translation but decreased CHIKV and SINV translation. Silencing of PCBP 1, on the other hand, decreased SFV translation.

In addition to hnRNPs, a number of host proteins have been shown to co-purify with the viral RNA and indicated to play role in the initiation of RNA synthesis. Short oligonucleotides corresponding to the 3' end of SINV, SFV, and RRV minus strand bind cellular proteins, and these might be needed for the initiation of the plus-strand RNA synthesis (Bruce et al., 2008; Bruce et al., 2010). Mosquito homolog of the La autoantigen binds to the 3' end of the SINV minus strands, and it may also play a role in the initiation of the plus-strand RNA synthesis as the La autoantigen is known to bind to the $3^{\prime}$ oligo(U) terminus of the transcripts made by RNA polymerase III and to control transcription termination as well as reinitiation by the polymerase (Buchholz et al., 1999; Chen and Ahlquist, 2000). In addition, mutations in the 5' cis elements or 3' UTR of the SINV genome have host-specific effects indicating that these elements interact with cellular factors (Chen et al., 2001; Frolov et al., 2001; Gorchakov et al., 2004; Niesters and Strauss, 1990a, b). It has been speculated that alphaviruses use PABP in the genome circularization and thus in the initiation of the minus-strand RNA synthesis, and PABP has been detected in the nsP2-containing complexes isolated from SINV-infected cells (Atasheva et al., 2007; Frolov et al., 2001). However, interaction between the poly(A) tail of 
the genome and PABP remains to be studied. In addition to the initiation, host proteins might be important in the switch from the minus- to plus-strand RNA synthesis. It has been suggested that RNase $L$ has a role in the shutoff of the minus-strand RNA synthesis and formation of stable RCs with transcriptional activity as knockout of RNase L results in continuous synthesis of the SINV minus- and plus-strand RNA (Sawicki et al., 2003).

To date, no host proteins has been shown to directly interact with the alphavirus polymerase and affect its activity. Once the structure of the RCs is resolved, we will be able to understand which host proteins play the most important roles in the function of the polymerase and the entire RC.

\section{Antivirals and alphavirus polymerase complex}

CHIKV and related alphaviruses cause febrile illness accompanied by myalgia, arthralgia, and rash. Although mortality is low, symptoms can last from weeks to months, or even years (Weaver and Lecuit, 2015). Recent emergence of CHIKV in the Caribbean was followed by epidemics (Leparc-Goffart et al., 2014) exemplifying the threat that vector-borne alphaviruses currently have on human health. Consequently, most of the antiviral work on alphaviruses has focused on CHIKV. Nevertheless, there are no approved, effective antivirals or vaccines against any human alphavirus (Abdelnabi et al., 2015; Ahola et al., 2015). Here, we focus on those antivirals which may target viral nsPs or host factors interacting with them.

The RNA-dependent RNA polymerase, or the RC, offers a good target for antivirals, and there are several modified nucleosides against alphaviruses. Nucleoside analog ribavirin, which causes inhibition of genome replication by depletion of GTP pools, has antiviral activity against several alphaviruses (Briolant et al., 2004; Leyssen et al., 2006). However, 6-azauridine, which causes intracellular UTP depletion, is a more effective antiviral against CHIKV and SFV than ribavirin (Briolant et al., 2004; Rada and Dragun, 1977; Scholte et al., 2013) although ribavirin and interferon- $\alpha 2 b$ have a subsynergistic effect on both viruses (Briolant et al., 2004). Furthermore, combination of ribavirin and doxycycline, which is a semi-synthetic tetracycline antibiotic and predicted to bind to nsP2 protease active site and E2 glycoprotein, gives good antiviral effect on CHIKV by inhibiting both entry and replication (Rothan et al., 2015). Ribavirin 5'-sulfamate, which is a close analog of ribavirin, is also active against SFV but 
it is highly cytotoxic (Smee et al., 1988). Mycophenolic acid inhibits CHIKV replication, even more efficiently than ribavirin, and the most likely mechanism is also GTP-pool depletion (Khan et al., 2011; Scholte et al., 2013). A SINV mutant resistant to both mycophenolic acid and ribavirin has three amino acid changing mutations in nsP1 indicating that these compounds affect the guanine 7-methyltransferase activity of nsP1 (Scheidel and Stollar, 1991). In contrast, a Gly641 to Asp substitution in nsP2 or a Cys483 to Tyr substitution in nsP4 renders CHIKV resistant to ribavirin and at the same time increases replication fidelity (Coffey et al., 2011; Stapleford et al., 2015). In contrast to their in vivo effects, ribavirin, 6-aza-uridine, or mycophenolic acid have no measurable effects on the CHIKV in vitro RNA-synthesizing activity. It might be that these nucleoside analogs are unable to inhibit CHIKV replication in vitro as they affect cellular NTP pools and the in vitro assay contains NTPs supplied in excess. In addition, some of the drugs may not be converted to their active, phosphorylated form during the in vitro replication assay (Albulescu et al., 2014).

Besides the polymerase activity, nsP1 is a promising target of alphavirus antivirals. In addition to mycophenolic acid and ribavirin, 3-deaza-adenosine inhibits CHIKV replication and most likely affect nsP1 by interfering of S-adenosylmethionine-dependent methylation (De Clercq, 1998; Scholte et al., 2013). In addition to nucleotide analogs, a novel class of small molecules have been observed to inhibit CHIKV, and the resistant variants have a Pro34 to Ser substitution in nsP1. Using VEEV nsP1, the authors showed that these molecules inhibit the guanylylation activity of nsP1 (Delang et al., 2016; Gigante et al., 2014).

Favipiravir (T-705) is a broad-spectrum antiviral and inhibits RNA viruses most likely by competing with the incorporation of ATP and GTP (Furuta et al., 2013). Two mechanisms have been proposed; chain termination or lethal mutagenesis. Favipiravir has been shown to block replication of both New and Old World alphaviruses, including CHIKV, SFV, SINV, Eastern equine encephalitis virus, Western equine encephalitis virus, VEEV, ONNV, RRV, and BFV (Delang et al., 2014; Julander et al., 2009). In addition, defluorinated analogue of favipiravir, T-1105, has the same effect (Delang et al., 2014). Favipiravir inhibits CHIKV RNA synthesis, and correlation between CHIKV RNA amount and infectivity decrease indicates that the mechanism of action is different than the induction of lethal mutagenesis. Low-level favipiravir-resistant CHIKV variants have a Lys291 to Arg mutation in nsP4, and this lysine is highly conserved in positive-strand RNA viruses. This suggests that the target site of favipiravir is the well-conserved region of the RdRp (Delang et al., 2014). 
As mentioned in the previous section, CHIKV and SINV nsP4-containing complexes interact with HSP-90, and drugs against HSP-90 have been shown inhibit CHIKV replication

792 (Rathore et al., 2014). A virtual screening simulation was used to find compounds against CHIKV nsP2, and one of the hits targeting the central part of the protease active site inhibited CHIKV replication (Bassetto et al., 2013). Continuing from these lead compounds, Das et al. described a set of related compounds, some of which were shown both to inhibit the nsP2 protease as well as virus replication (Das et al., 2016). Collectively, the number of potential antivirals against alphaviruses has increased in recent years and it seems that the possibility to (pre)treat humans is soon at hand. Furthermore, recognition of host factors essential for the formation and function of the alphaviruses RCs will open more avenues to explore new antivirals.

\section{Concluding remarks}

In spite of the extensive studies on alphavirus replication strategies, the knowledge of the biochemical and structural properties of the viral polymerase nsP4 is surprisingly limited. This unusual polymerase apparently cannot act alone but needs the co-operation of and interaction with the other nonstructural proteins. Many types of experiments have given important information concerning the activities of both nsP4 and the other nsPs and defined their roles during RNA synthesis and replication complex assembly.

The RCs form membranous invaginations (spherules) on cellular membranes and are thought to restrict the replication to a protected environment inaccessible to cellular defence mechanisms. At the same time, spherules gather all the necessary components to a compact structure, which may limit the activities of the polymerase complex in important ways. However, very little is still known concerning the cellular proteins that may participate in spherule formation or function, and how and in what numbers both viral nonstructural proteins and host proteins are structurally arranged in the RCs. All these details would be invaluable in order to fully understand how different RNA species are produced, how the RC switches the strand-specificity and what the exact roles of each nonstructural protein within a spherule are. It has been shown that the polymerase activity together with the presence of other nsPs is necessary for spherule formation and that the spherule size is determined by the length of the RNA template (Kallio et al., 2013). Yet the chronological order of spherule 
821 formation steps, including the timing of RNA recruitment and complex assembly are 822 unresolved.

Alphaviruses are emergently causing epidemics on several continents, but there are no approved antivirals or vaccines available. The vulnerable replication steps involving both the nonstructural proteins and the participating host components would be ideal targets in the fight against these viruses, making it clear that more precise structural and functional knowledge of the RC is required.

\section{Acknowledgements}

830 Giuseppe Balistreri and Helena Vihinen are acknowledged for the reconstructed electron microscopy image of a spherule (Fig. 3C) and Katri Kallio is thanked for her help with the drawings. This work was supported by Academy of Finland grants 265997 (to TA) and 274748 833 (to MKP).

Table 1. Summary of the viruses discussed in this review.

\begin{tabular}{|l|l|l|}
\hline Genus / Family & Virus species & Abbreviation \\
\hline Alphavirus / Togaviridae & Barmah Forest virus & BFV \\
\cline { 2 - 3 } & Chikungunya virus & CHIKV \\
\cline { 2 - 3 } & Eastern equine encephalitis virus & EEEV \\
\cline { 2 - 3 } & O'nyong-nyong virus & ONNV \\
\cline { 2 - 3 } & Ross River virus & RRV \\
\cline { 2 - 3 } & Semliki Forest virus & SFV \\
\cline { 2 - 3 } & Sindbis virus & SINV \\
\cline { 2 - 3 } & Venezuelan equine encephalitis virus & VEEV \\
\cline { 2 - 3 } & Western equine encephalitis virus & WEEV \\
\hline Rubivirus / Togaviridae & Rubella virus & RUBV \\
\hline Bromovirus / Bromoviridae & Brome mosaic virus & BMV \\
\hline Alphanodavirus / Nodaviridae & Flock House virus & FHV \\
\hline
\end{tabular}




\section{Figure legends}

\section{Figure 1.}

840 Alphavirus genome structure. The positive-sense RNA genome is about 11,5 kilobases in 841 length and contains two open reading frames; first encoding for the nonstructural proteins 842 (nsPs) 1-4 and second for structural proteins (C, capsid; E1/2/3, envelope glycoproteins and $8436 \mathrm{~K}$, a $6 \mathrm{kDa}$ protein). UTR, untranslated region; SGP, subgenomic promoter; $\mathrm{A}(\mathrm{n})$, polyA.

Figure 2.

846 Schematic of the polyprotein processing and RNA synthesis. After disassembly of the 847 incoming virus particles, the viral plus-strand RNA is released and the nonstructural proteins are translated as a polyprotein. After cleavage of nsP4, the RC synthesizes minus strand from the genomic RNA. Further cleavage of all nsPs to individual proteins switches synthesis to genomic and subgenomic positive-strand RNA. The structural proteins are translated from subgenomic RNA, leading to the packaging of viral genomic RNA to the forming nucleocapsid.

Figure 3.

Membranous replication complexes of SFV. A) Spherules located at the plasma membrane at an early time point. B) Type I cytopathic vacuole (CPV-I) of an infected cell, containing numerous spherules lining the membrane. C) 3D reconstruction of a single spherule. D) Schematic of a spherule with replication complex proteins located hypothetically on the neck region and newly synthesized RNA coming out. The scale bars in A and B are $200 \mathrm{~nm}$ and 100 $\mathrm{nm}$, respectively.

Figure 4.

862 A schematic model of the replicative form (RF) and replicative intermediate (RI). Purple

863 indicates the polymerase complex. RI contains several unfinished plus strands. Minus strands 864 are shown as blue and plus strands as pink.

Figure 5.

867 Examples of host proteins identified in alphavirus nsP-containing complexes. nsP1-, nsP2-, nsP3- and nsP4-containing complexes and their interactions with membrane-curvature 
proteins amphiphysins, poly(A)-binding protein (PABP), heterogeneous nuclear and HSP-90, Ras-GTPase-activating protein (SH3 domain)-binding proteins G3BP1 and 2, and Sphingosine kinase 2 (SK2).

\section{References}

Abdelnabi, R., Neyts, J., Delang, L., 2015. Towards antivirals against chikungunya virus. Antiviral Res 121, 59-68.

Adkins, S., Stawicki, S.S., Faurote, G., Siegel, R.W., Kao, C.C., 1998. Mechanistic analysis of RNA synthesis by RNA-dependent RNA polymerase from two promoters reveals similarities to DNAdependent RNA polymerase. RNA 4, 455-470. 1273.

Ahlquist, P., 2006. Parallels among positive-strand RNA viruses, reverse-transcribing viruses and double-stranded RNA viruses. Nature reviews. Microbiology 4, 371-382.

Ahlquist, P., Noueiry, A.O., Lee, W.M., Kushner, D.B., Dye, B.T., 2003. Host factors in positive-strand RNA virus genome replication. J Virol 77, 8181-8186.

Ahola, T., Couderc, T., Ng, L.F., Hallengard, D., Powers, A., Lecuit, M., Esteban, M., Merits, A., Roques, P., Liljestrom, P., 2015. Therapeutics and vaccines against chikungunya virus. Vector Borne Zoonotic Dis 15, 250-257.

Ahola, T., Kujala, P., Tuittila, M., Blom, T., Laakkonen, P., Hinkkanen, A., Auvinen, P., 2000. Effects of palmitoylation of replicase protein nsP1 on alphavirus infection. J Virol 74, 6725-6733.

Ahola, T., Kääriäinen, L., 1995. Reaction in alphavirus mRNA capping: formation of a covalent complex of nonstructural protein nsP1 with 7-methyl-GMP. Proc Natl Acad Sci U S A 92, 507-511. Ahola, T., Lampio, A., Auvinen, P., Kääriäinen, L., 1999. Semliki Forest virus mRNA capping enzyme requires association with anionic membrane phospholipids for activity. EMBO J 18, 3164-3172. Albulescu, I.C., Tas, A., Scholte, F.E., Snijder, E.J., van Hemert, M.J., 2014. An in vitro assay to study chikungunya virus RNA synthesis and the mode of action of inhibitors. J Gen Virol 95, 2683-2692. Atasheva, S., Gorchakov, R., English, R., Frolov, I., Frolova, E., 2007. Development of Sindbis viruses encoding nsP2/GFP chimeric proteins and their application for studying nsP2 functioning. J Virol 81, 5046-5057.

Balistreri, G., Caldentey, J., Kaariainen, L., Ahola, T., 2007. Enzymatic defects of the nsP2 proteins of Semliki Forest virus temperature-sensitive mutants. J Virol 81, 2849-2860.

Barton, D.J., Sawicki, S.G., Sawicki, D.L., 1988. Demonstration in vitro of temperature-sensitive elongation of RNA in Sindbis virus mutant ts6. J Virol 62, 3597-3602.

Barton, D.J., Sawicki, S.G., Sawicki, D.L., 1991. Solubilization and immunoprecipitation of alphavirus replication complexes. J Virol 65, 1496-1506.

Bassetto, M., De Burghgraeve, T., Delang, L., Massarotti, A., Coluccia, A., Zonta, N., Gatti, V., Colombano, G., Sorba, G., Silvestri, R., Tron, G.C., Neyts, J., Leyssen, P., Brancale, A., 2013. Computer-aided identification, design and synthesis of a novel series of compounds with selective antiviral activity against chikungunya virus. Antiviral Res 98, 12-18.

Briolant, S., Garin, D., Scaramozzino, N., Jouan, A., Crance, J.M., 2004. In vitro inhibition of Chikungunya and Semliki Forest viruses replication by antiviral compounds: synergistic effect of interferon-alpha and ribavirin combination. Antiviral Res 61, 111-117.

Bruce, J.W., Ahlquist, P., Young, J.A., 2008. The host cell sulfonation pathway contributes to retroviral infection at a step coincident with provirus establishment. PLoS Pathog 4, e1000207. 
Bruce, J.W., Hierl, M., Young, J.A., Ahlquist, P., 2010. Cellular transcription factor ZASC1 regulates murine leukemia virus transcription. J Virol 84, 7473-7483.

Buchholz, U.J., Finke, S., Conzelmann, K.K., 1999. Generation of bovine respiratory syncytial virus (BRSV) from cDNA: BRSV NS2 is not essential for virus replication in tissue culture, and the human RSV leader region acts as a functional BRSV genome promoter. J Virol 73, 251-259.

Chen, J., Ahlquist, P., 2000. Brome mosaic virus polymerase-like protein $2 \mathrm{a}$ is directed to the endoplasmic reticulum by helicase-like viral protein 1a. J Virol 74, 4310-4318.

Chen, J., Noueiry, A., Ahlquist, P., 2001. Brome mosaic virus Protein 1a recruits viral RNA2 to RNA replication through a 5' proximal RNA2 signal. J Virol 75, 3207-3219.

Clewley, J.P., Kennedy, S.I., 1976. Purification and polypeptide composition of Semliki Forest virus RNA polymerase. J Gen Virol 32, 395-411.

Coffey, L.L., Beeharry, Y., Borderia, A.V., Blanc, H., Vignuzzi, M., 2011. Arbovirus high fidelity variant loses fitness in mosquitoes and mice. Proc Natl Acad Sci U S A 108, 16038-16043.

Cristea, I.M., Carroll, J.W., Rout, M.P., Rice, C.M., Chait, B.T., MacDonald, M.R., 2006. Tracking and elucidating Alphavirus-host protein interactions. J Biol Chem 281, 30269-30278.

Cristea, I.M., Rozjabek, H., Molloy, K.R., Karki, S., White, L.L., Rice, C.M., Rout, M.P., Chait, B.T., MacDonald, M.R., 2010. Host factors associated with the Sindbis virus RNA-dependent RNA polymerase: role for G3BP1 and G3BP2 in virus replication. J Virol 84, 6720-6732.

Cross, R.K., Gomatos, P.J., 1981. Concomitant methylation and synthesis in vitro of Semliki Forest virus (SFV) ss RNAs by a fraction from infected cells. Virology 114, 542-554.

Das, P.K., Merits, A., Lulla, A., 2014. Functional crosstalk between distant domains of Chikungunya virus non-structural protein 2 is decisive for its RNA-modulating activity. J Biol Chem 289, 5635-5653. Das, P.K., Puusepp, L., Varghese, F.S., Utt, A., Ahola, T., Kananovich, D.G., Lopp, M., Merits, A., Karelson, M., 2016. Design and Validation of Novel Chikungunya Virus Protease Inhibitors. Antimicrob Agents Chemother 60, 7382-7395.

De Clercq, E., 1998. Carbocyclic adenosine analogues as S-adenosylhomocysteine hydrolase inhibitors and antiviral agents: recent advances. Nucleosides Nucleotides 17, 625-634.

de Groot, R.J., Hardy, W.R., Shirako, Y., Strauss, J.H., 1990. Cleavage-site preferences of Sindbis virus polyproteins containing the non-structural proteinase. Evidence for temporal regulation of polyprotein processing in vivo. EMBO J 9, 2631-2638.

de Groot, R.J., Rumenapf, T., Kuhn, R.J., Strauss, E.G., Strauss, J.H., 1991. Sindbis virus RNA polymerase is degraded by the N-end rule pathway. Proc Natl Acad Sci U S A 88, 8967-8971. Delang, L., Li, C., Tas, A., Querat, G., Albulescu, I.C., De Burghgraeve, T., Guerrero, N.A., Gigante, A., Piorkowski, G., Decroly, E., Jochmans, D., Canard, B., Snijder, E.J., Perez-Perez, M.J., van Hemert, M.J., Coutard, B., Leyssen, P., Neyts, J., 2016. The viral capping enzyme nsP1: a novel target for the inhibition of chikungunya virus infection. Sci Rep 6, 31819.

Delang, L., Segura Guerrero, N., Tas, A., Querat, G., Pastorino, B., Froeyen, M., Dallmeier, K., Jochmans, D., Herdewijn, P., Bello, F., Snijder, E.J., de Lamballerie, X., Martina, B., Neyts, J., van Hemert, M.J., Leyssen, P., 2014. Mutations in the chikungunya virus non-structural proteins cause resistance to favipiravir (T-705), a broad-spectrum antiviral. J Antimicrob Chemother 69, 2770-2784. Deo, R.C., Bonanno, J.B., Sonenberg, N., Burley, S.K., 1999. Recognition of polyadenylate RNA by the poly(A)-binding protein. Cell 98, 835-845.

Fata, C.L., Sawicki, S.G., Sawicki, D.L., 2002a. Alphavirus minus-strand RNA synthesis: identification of a role for Arg183 of the nsP4 polymerase. J Virol 76, 8632-8640.

Fata, C.L., Sawicki, S.G., Sawicki, D.L., 2002b. Modification of Asn374 of nsP1 suppresses a Sindbis virus nsP4 minus-strand polymerase mutant. J Virol 76, 8641-8649.

Forrester, N.L., Guerbois, M., Seymour, R.L., Spratt, H., Weaver, S.C., 2012. Vector-borne transmission imposes a severe bottleneck on an RNA virus population. PLoS Pathog 8, e1002897. Foy, N.J., Akhrymuk, M., Akhrymuk, I., Atasheva, S., Bopda-Waffo, A., Frolov, I., Frolova, E.I., 2013. Hypervariable domains of nsP3 proteins of New World and Old World alphaviruses mediate formation of distinct, virus-specific protein complexes. J Virol 87, 1997-2010. 
967 Frey, T.K., Strauss, J.H., 1978. Replication of Sindbis virus. VI. Poly(A) and poly(U) in virus-specific

968 RNA species. Virology 86, 494-506.

969 Friedman, R.M., Levin, J.G., Grimley, P.M., Berezesky, I.K., 1972. Membrane-associated replication

970 complex in arbovirus infection. J Virol 10, 504-515.

971 Frolov, I., Hardy, R., Rice, C.M., 2001. Cis-acting RNA elements at the 5 ' end of Sindbis virus genome

972 RNA regulate minus- and plus-strand RNA synthesis. RNA 7, 1638-1651.

973 Frolova, E., Gorchakov, R., Garmashova, N., Atasheva, S., Vergara, L.A., Frolov, I., 2006. Formation of

974 nsP3-specific protein complexes during Sindbis virus replication. J Virol 80, 4122-4134.

975

976

977

978

979

980

981

982

983 Frolova, E.I., Gorchakov, R., Pereboeva, L., Atasheva, S., Frolov, I., 2010. Functional Sindbis virus replicative complexes are formed at the plasma membrane. J Virol 84, 11679-11695.

Fros, J.J., Domeradzka, N.E., Baggen, J., Geertsema, C., Flipse, J., Vlak, J.M., Pijlman, G.P., 2012. Chikungunya virus nsP3 blocks stress granule assembly by recruitment of G3BP into cytoplasmic foci. J Virol 86, 10873-10879.

Froshauer, S., Kartenbeck, J., Helenius, A., 1988. Alphavirus RNA replicase is located on the cytoplasmic surface of endosomes and lysosomes. The Journal of cell biology 107, 2075-2086. Furuta, Y., Gowen, B.B., Takahashi, K., Shiraki, K., Smee, D.F., Barnard, D.L., 2013. Favipiravir (T-705), a novel viral RNA polymerase inhibitor. Antiviral Res 100, 446-454.

Gigante, A., Canela, M.D., Delang, L., Priego, E.M., Camarasa, M.J., Querat, G., Neyts, J., Leyssen, P., Perez-Perez, M.J., 2014. Identification of [1,2,3]triazolo[4,5-d]pyrimidin-7(6H)-ones as novel inhibitors of Chikungunya virus replication. J Med Chem 57, 4000-4008.

Gomatos, P.J., Kääriäinen, L., Keränen, S., Ranki, M., Sawicki, D.L., 1980. Semliki Forest virus replication complex capable of synthesizing 42S and 26S nascent RNA chains. J Gen Virol 49, 61-69. Gorchakov, R., Frolova, E., Sawicki, S., Atasheva, S., Sawicki, D., Frolov, I., 2008a. A new role for ns polyprotein cleavage in Sindbis virus replication. J Virol 82, 6218-6231.

Gorchakov, R., Garmashova, N., Frolova, E., Frolov, I., 2008b. Different types of nsP3-containing protein complexes in Sindbis virus-infected cells. J Virol 82, 10088-10101.

Gorchakov, R., Hardy, R., Rice, C.M., Frolov, I., 2004. Selection of functional 5' cis-acting elements promoting efficient sindbis virus genome replication. J Virol 78, 61-75.

Graham, T.R., Kozlov, M.M., 2010. Interplay of proteins and lipids in generating membrane curvature. Curr Opin Cell Biol 22, 430-436.

Grimley, P.M., Berezesky, I.K., Friedman, R.M., 1968. Cytoplasmic structures associated with an arbovirus infection: loci of viral ribonucleic acid synthesis. J Virol 2, 1326-1338.

Grimley, P.M., Levin, J.G., Berezesky, I.K., Friedman, R.M., 1972. Specific membranous structures associated with the replication of group A arboviruses. J Virol 10, 492-503.

Gui, H., Lu, C.W., Adams, S., Stollar, V., Li, M.L., 2010. hnRNP A1 interacts with the genomic and subgenomic RNA promoters of Sindbis virus and is required for the synthesis of $G$ and SG RNA. J Biomed Sci 17, 59.

Hahn, Y.S., Grakoui, A., Rice, C.M., Strauss, E.G., Strauss, J.H., 1989a. Mapping of RNA- temperaturesensitive mutants of Sindbis virus: complementation group F mutants have lesions in nsP4. J Virol 63, 1194-1202.

Hahn, Y.S., Strauss, E.G., Strauss, J.H., 1989b. Mapping of RNA- temperature-sensitive mutants of Sindbis virus: assignment of complementation groups $A, B$, and $G$ to nonstructural proteins. J Virol 63, 3142-3150.

Hajjou, M., Hill, K.R., Subramaniam, S.V., Hu, J.Y., Raju, R., 1996. Nonhomologous RNA-RNA recombination events at the $3^{\prime}$ nontranslated region of the Sindbis virus genome: hot spots and utilization of nonviral sequences. J Virol 70, 5153-5164.

Hardy, R.W., 2006. The role of the 3 ' terminus of the Sindbis virus genome in minus-strand initiation site selection. Virology 345, 520-531.

1016 Hardy, R.W., Rice, C.M., 2005. Requirements at the 3 ' end of the sindbis virus genome for efficient synthesis of minus-strand RNA. J Virol 79, 4630-4639. 
Hardy, W.R., Hahn, Y.S., de Groot, R.J., Strauss, E.G., Strauss, J.H., 1990. Synthesis and processing of the nonstructural polyproteins of several temperature-sensitive mutants of Sindbis virus. Virology 177, 199-208.

Hardy, W.R., Strauss, J.H., 1988. Processing the nonstructural polyproteins of Sindbis virus: study of the kinetics in vivo by using monospecific antibodies. J Virol 62, 998-1007.

Hardy, W.R., Strauss, J.H., 1989. Processing the nonstructural polyproteins of sindbis virus: nonstructural proteinase is in the C-terminal half of nsP2 and functions both in cis and in trans. J Virol 63, 4653-4664.

Hellström, K., Kallio, K., Meriläinen, H.M., Jokitalo, E., Ahola, T., 2016. Ability of minus strands and modified plus strands to act as templates in Semliki Forest virus RNA replication. J Gen Virol 97, 1395-1407.

Hill, K.R., Hajjou, M., Hu, J.Y., Raju, R., 1997. RNA-RNA recombination in Sindbis virus: roles of the 3' conserved motif, poly(A) tail, and nonviral sequences of template RNAs in polymerase recognition and template switching. J Virol 71, 2693-2704.

Jose, J., Snyder, J.E., Kuhn, R.J., 2009. A structural and functional perspective of alphavirus replication and assembly. Future Microbiol 4, 837-856.

Julander, J.G., Smee, D.F., Morrey, J.D., Furuta, Y., 2009. Effect of T-705 treatment on western equine encephalitis in a mouse model. Antiviral Res 82, 169-171.

Kääriäinen, L., Ahola, T., 2002. Functions of alphavirus nonstructural proteins in RNA replication. Progress in nucleic acid research and molecular biology 71, 187-222.

Kallio, K., Hellström, K., Balistreri, G., Spuul, P., Jokitalo, E., Ahola, T., 2013. Template RNA length determines the size of replication complex spherules for Semliki Forest virus. J Virol 87, 9125-9134. Kallio, K., Hellström, K., Jokitalo, E., Ahola, T., 2016. RNA Replication and Membrane Modification Require the Same Functions of Alphavirus Nonstructural Proteins. J Virol 90, 1687-1692.

Keränen, S., Kääriäinen, L., 1979. Functional defects of RNA-negative temperature-sensitive mutants of Sindbis and Semliki Forest viruses. J Virol 32, 19-29.

Khan, A.H., Morita, K., Parquet Md Mdel, C., Hasebe, F., Mathenge, E.G., Igarashi, A., 2002. Complete nucleotide sequence of chikungunya virus and evidence for an internal polyadenylation site. J Gen Virol 83, 3075-3084.

Khan, M., Dhanwani, R., Patro, I.K., Rao, P.V., Parida, M.M., 2011. Cellular IMPDH enzyme activity is a potential target for the inhibition of Chikungunya virus replication and virus induced apoptosis in cultured mammalian cells. Antiviral Res 89, 1-8.

Kim, D.Y., Reynaud, J.M., Rasalouskaya, A., Akhrymuk, I., Mobley, J.A., Frolov, I., Frolova, E.I., 2016. New World and Old World Alphaviruses Have Evolved to Exploit Different Components of Stress Granules, FXR and G3BP Proteins, for Assembly of Viral Replication Complexes. PLoS Pathog 12, e1005810.

Koonin, E.V., Dolja, V.V., Krupovic, M., 2015. Origins and evolution of viruses of eukaryotes: The ultimate modularity. Virology 479-480, 2-25.

Kujala, P., Ahola, T., Ehsani, N., Auvinen, P., Vihinen, H., Kääriäinen, L., 1999. Intracellular distribution of rubella virus nonstructural protein P150. J Virol 73, 7805-7811.

Kujala, P., Ikaheimonen, A., Ehsani, N., Vihinen, H., Auvinen, P., Kääriäinen, L., 2001. Biogenesis of the Semliki Forest virus RNA replication complex. J Virol 75, 3873-3884.

Laakkonen, P., Ahola, T., Kääriäinen, L., 1996. The effects of palmitoylation on membrane association of Semliki forest virus RNA capping enzyme. J Biol Chem 271, 28567-28571.

Lampio, A., Kilpeläinen, I., Pesonen, S., Karhi, K., Auvinen, P., Somerharju, P., Kääriäinen, L., 2000. Membrane binding mechanism of an RNA virus-capping enzyme. J Biol Chem 275, 37853-37859. Lemm, J.A., Bergqvist, A., Read, C.M., Rice, C.M., 1998. Template-dependent initiation of Sindbis virus RNA replication in vitro. J Virol 72, 6546-6553.

Lemm, J.A., Rice, C.M., 1993a. Assembly of functional Sindbis virus RNA replication complexes: requirement for coexpression of P123 and P34. J Virol 67, 1905-1915. 
Lemm, J.A., Rice, C.M., 1993b. Roles of nonstructural polyproteins and cleavage products in

1068 regulating Sindbis virus RNA replication and transcription. J Virol 67, 1916-1926.

1069 Lemm, J.A., Rumenapf, T., Strauss, E.G., Strauss, J.H., Rice, C.M., 1994. Polypeptide requirements for 1070 assembly of functional Sindbis virus replication complexes: a model for the temporal regulation of 1071 minus- and plus-strand RNA synthesis. EMBO J 13, 2925-2934.

1072 Leparc-Goffart, I., Nougairede, A., Cassadou, S., Prat, C., de Lamballerie, X., 2014. Chikungunya in the 1073 Americas. Lancet 383, 514.

1074 Levis, R., Schlesinger, S., Huang, H.V., 1990. Promoter for Sindbis virus RNA-dependent subgenomic RNA transcription. J Virol 64, 1726-1733.

Leyssen, P., De Clercq, E., Neyts, J., 2006. The anti-yellow fever virus activity of ribavirin is independent of error-prone replication. Mol Pharmacol 69, 1461-1467.

Li, C., Debing, Y., Jankevicius, G., Neyts, J., Ahel, I., Coutard, B., Canard, B., 2016. Viral Macro Domains Reverse Protein ADP-Ribosylation. J Virol 90, 8478-8486.

Li, G., Rice, C.M., 1993. The signal for translational readthrough of a UGA codon in Sindbis virus RNA involves a single cytidine residue immediately downstream of the termination codon. J Virol 67, 5062-5067.

Li, M.L., Kwan, T.Y., Simmonds, H.A., Stollar, V., 2008. Synthesis of genomic and subgenomic RNA in mosquito cells infected with two Sindbis virus nsP4 mutants: influence of intracellular nucleoside triphosphate concentrations. J Virol 82, 6880-6888.

Li, M.L., Lin, Y.H., Simmonds, H.A., Stollar, V., 2004. A mutant of Sindbis virus which is able to replicate in cells with reduced CTP makes a replicase/transcriptase with a decreased Km for CTP. J Virol 78, 9645-9651.

Li, M.L., Lin, Y.H., Stollar, V., 2005. A cell-free system for the synthesis of Sindbis virus subgenomic RNA: importance of the concentration of the initiating NTP. Virology 341, 24-33.

1091 Li, M.L., Stollar, V., 2004. Identification of the amino acid sequence in Sindbis virus nsP4 that binds to 1092 the promoter for the synthesis of the subgenomic RNA. Proc Natl Acad Sci U S A 101, 9429-9434.

1093 Li, M.L., Stollar, V., 2007. Distinct sites on the Sindbis virus RNA-dependent RNA polymerase for 1094 binding to the promoters for the synthesis of genomic and subgenomic RNA. J Virol 81, 4371-4373. Li, M.L., Wang, H., Stollar, V., 2010. In vitro synthesis of Sindbis virus genomic and subgenomic RNAs: influence of nsP4 mutations and nucleoside triphosphate concentrations. J Virol 84, 2732-2739. Lin, Y.H., Simmonds, H.A., Stollar, V., 2002. Restriction of a Sindbis virus mutant in BHK cells and relief of the restriction by the addition of adenosine. Virology 292, 78-86.

Lin, Y.H., Yadav, P., Ravatn, R., Stollar, V., 2000. A mutant of Sindbis virus that is resistant to pyrazofurin encodes an altered RNA polymerase. Virology 272, 61-71.

Lorizate, M., Krausslich, H.G., 2011. Role of lipids in virus replication. Cold Spring Harb Perspect Biol 3, a004820.

Lulla, V., Sawicki, D.L., Sawicki, S.G., Lulla, A., Merits, A., Ahola, T., 2008. Molecular defects caused by temperature-sensitive mutations in Semliki Forest virus nsP1. J Virol 82, 9236-9244.

Michel, M.R., Gomatos, P.J., 1973. Semliki forest virus-specific RNAs synthesized in vitro by enzyme from infected BHK cells. J Virol 11, 900-914.

Miller, S., Krijnse-Locker, J., 2008. Modification of intracellular membrane structures for virus replication. Nature reviews. Microbiology 6, 363-374.

Monroe, S.S., Schlesinger, S., 1983. RNAs from two independently isolated defective interfering particles of Sindbis virus contain a cellular tRNA sequence at their 5 ' ends. Proc Natl Acad Sci U S A 80, 3279-3283.

Monroe, S.S., Schlesinger, S., 1984. Common and distinct regions of defective-interfering RNAs of Sindbis virus. J Virol 49, 865-872.

Mönttinen, H.A., Ravantti, J.J., Stuart, D.I., Poranen, M.M., 2014. Automated structural comparisons clarify the phylogeny of the right-hand-shaped polymerases. Mol Biol Evol 31, 2741-2752.

Neufeldt, C.J., Joyce, M.A., Van Buuren, N., Levin, A., Kirkegaard, K., Gale, M., Jr., Tyrrell, D.L., Wozniak, R.W., 2016. The Hepatitis C Virus-Induced Membranous Web and Associated Nuclear 
Transport Machinery Limit Access of Pattern Recognition Receptors to Viral Replication Sites. PLoS Pathog 12, e1005428.

Neuvonen, M., Kazlauskas, A., Martikainen, M., Hinkkanen, A., Ahola, T., Saksela, K., 2011. SH3 domain-mediated recruitment of host cell amphiphysins by alphavirus nsP3 promotes viral RNA replication. PLoS Pathog 7, e1002383.

Nickens, D.G., Hardy, R.W., 2008. Structural and functional analyses of stem-loop 1 of the Sindbis virus genome. Virology 370, 158-172.

Niesters, H.G., Strauss, J.H., 1990a. Defined mutations in the 5 ' nontranslated sequence of Sindbis virus RNA. J Virol 64, 4162-4168.

Niesters, H.G., Strauss, J.H., 1990b. Mutagenesis of the conserved 51-nucleotide region of Sindbis virus. J Virol 64, 1639-1647.

Nikonov, A., Molder, T., Sikut, R., Kiiver, K., Mannik, A., Toots, U., Lulla, A., Lulla, V., Utt, A., Merits, A., Ustav, M., 2013. RIG-I and MDA-5 detection of viral RNA-dependent RNA polymerase activity restricts positive-strand RNA virus replication. PLoS Pathog 9, e1003610.

O'Reilly, E.K., Kao, C.C., 1998. Analysis of RNA-dependent RNA polymerase structure and function as guided by known polymerase structures and computer predictions of secondary structure. Virology 252, 287-303.

Ou, J.H., Strauss, E.G., Strauss, J.H., 1983. The 5'-terminal sequences of the genomic RNAs of several alphaviruses. J Mol Biol 168, 1-15.

Panas, M.D., Ahola, T., Mclnerney, G.M., 2014. The C-terminal repeat domains of nsP3 from the Old World alphaviruses bind directly to G3BP. J Virol 88, 5888-5893.

Panas, M.D., Varjak, M., Lulla, A., Eng, K.E., Merits, A., Karlsson Hedestam, G.B., McInerney, G.M., 2012. Sequestration of G3BP coupled with efficient translation inhibits stress granules in Semliki Forest virus infection. Mol Biol Cell 23, 4701-4712.

Paul, D., Bartenschlager, R., 2013. Architecture and biogenesis of plus-strand RNA virus replication factories. World journal of virology 2, 32-48.

Peersen, O.B., 2017. Picornaviral polymerase structure, function and fidelity modulation. Virus Res. Peränen, J., Laakkonen, P., Hyvonen, M., Kääriäinen, L., 1995. The alphavirus replicase protein nsP1 is membrane-associated and has affinity to endocytic organelles. Virology 208, 610-620.

Peränen, J., Rikkonen, M., Liljestrom, P., Kääriäinen, L., 1990. Nuclear localization of Semliki Forest virus-specific nonstructural protein nsP2. J Virol 64, 1888-1896.

Peränen, J., Takkinen, K., Kalkkinen, N., Kääriäinen, L., 1988. Semliki Forest virus-specific nonstructural protein nsP3 is a phosphoprotein. J Gen Virol 69 ( Pt 9), 2165-2178.

Pflug, A., Lukarska, M., Resa-Infante, P., Reich, S., Cusack, S., 2017. Structural insights into RNA synthesis by the influenza virus transcription replication machine. Virus Res.

Poirier, E.Z., Mounce, B.C., Rozen-Gagnon, K., Hooikaas, P.J., Stapleford, K.A., Moratorio, G., Vignuzzi, M., 2016. Low-Fidelity Polymerases of Alphaviruses Recombine at Higher Rates To Overproduce Defective Interfering Particles. J Virol 90, 2446-2454.

Rada, B., Dragun, M., 1977. Antiviral action and selectivity of 6-azauridine. Ann N Y Acad Sci 284, 410-417.

Raju, R., Hajjou, M., Hill, K.R., Botta, V., Botta, S., 1999. In vivo addition of poly(A) tail and AU-rich sequences to the $3^{\prime}$ terminus of the Sindbis virus RNA genome: a novel 3 '-end repair pathway. J Virol 73, 2410-2419.

Raju, R., Subramaniam, S.V., Hajjou, M., 1995. Genesis of Sindbis virus by in vivo recombination of nonreplicative RNA precursors. J Virol 69, 7391-7401.

Ranki, M., Kääriäinen, L., 1979. Solubilized RNA replication complex from Semliki Forest virusinfected cells. Virology 98, 298-307.

Rathore, A.P., Haystead, T., Das, P.K., Merits, A., Ng, M.L., Vasudevan, S.G., 2014. Chikungunya virus nsP3 \& nsP4 interacts with HSP-90 to promote virus replication: HSP-90 inhibitors reduce CHIKV infection and inflammation in vivo. Antiviral Res 103, 7-16. 
Reid, C.R., Airo, A.M., Hobman, T.C., 2015a. The Virus-Host Interplay: Biogenesis of +RNA Replication Complexes. Viruses 7, 4385-4413.

Reid, S.P., Tritsch, S.R., Kota, K., Chiang, C.Y., Dong, L., Kenny, T., Brueggemann, E.E., Ward, M.D., Cazares, L.H., Bavari, S., 2015b. Sphingosine kinase 2 is a chikungunya virus host factor co-localized with the viral replication complex. Emerg Microbes Infect 4, e61.

Rikkonen, M., Peränen, J., Kääriäinen, L., 1992. Nuclear and nucleolar targeting signals of Semliki Forest virus nonstructural protein nsP2. Virology 189, 462-473.

Romero-Brey, I., Bartenschlager, R., 2014. Membranous replication factories induced by plus-strand RNA viruses. Viruses 6, 2826-2857.

Rothan, H.A., Bahrani, H., Mohamed, Z., Teoh, T.C., Shankar, E.M., Rahman, N.A., Yusof, R., 2015. A combination of doxycycline and ribavirin alleviated chikungunya infection. PLoS One 10, e0126360. Rozen-Gagnon, K., Stapleford, K.A., Mongelli, V., Blanc, H., Failloux, A.B., Saleh, M.C., Vignuzzi, M., 2014. Alphavirus mutator variants present host-specific defects and attenuation in mammalian and insect models. PLoS Pathog 10, e1003877.

Rubach, J.K., Wasik, B.R., Rupp, J.C., Kuhn, R.J., Hardy, R.W., Smith, J.L., 2009. Characterization of purified Sindbis virus nsP4 RNA-dependent RNA polymerase activity in vitro. Virology 384, 201-208. Rupp, J.C., Jundt, N., Hardy, R.W., 2011. Requirement for the amino-terminal domain of sindbis virus nsP4 during virus infection. J Virol 85, 3449-3460.

Rupp, J.C., Sokoloski, K.J., Gebhart, N.N., Hardy, R.W., 2015. Alphavirus RNA synthesis and nonstructural protein functions. J Gen Virol 96, 2483-2500.

Salonen, A., Ahola, T., Kääriäinen, L., 2005. Viral RNA replication in association with cellular membranes. Current topics in microbiology and immunology 285, 139-173.

Salonen, A., Vasiljeva, L., Merits, A., Magden, J., Jokitalo, E., Kääriäinen, L., 2003. Properly folded nonstructural polyprotein directs the semliki forest virus replication complex to the endosomal compartment. J Virol 77, 1691-1702.

Sawicki, D., Barkhimer, D.B., Sawicki, S.G., Rice, C.M., Schlesinger, S., 1990. Temperature sensitive shut-off of alphavirus minus strand RNA synthesis maps to a nonstructural protein, nsP4. Virology 174, 43-52.

Sawicki, D.L., Gomatos, P.J., 1976. Replication of semliki forest virus: polyadenylate in plus-strand RNA and polyuridylate in minus-strand RNA. J Virol 20, 446-464.

Sawicki, D.L., Sawicki, S.G., 1980. Short-lived minus-strand polymerase for Semliki Forest virus. J Virol 34, 108-118.

Sawicki, D.L., Sawicki, S.G., 1985. Functional analysis of the A complementation group mutants of Sindbis HR virus. Virology 144, 20-34.

Sawicki, D.L., Sawicki, S.G., 1993. A second nonstructural protein functions in the regulation of alphavirus negative-strand RNA synthesis. J Virol 67, 3605-3610.

Sawicki, D.L., Sawicki, S.G., Keränen, S., Kääriäinen, L., 1981. Specific Sindbis virus-coded function for minus-strand RNA synthesis. J Virol 39, 348-358.

Sawicki, D.L., Silverman, R.H., Williams, B.R., Sawicki, S.G., 2003. Alphavirus minus-strand synthesis and persistence in mouse embryo fibroblasts derived from mice lacking RNase $L$ and protein kinase R. J Virol 77, 1801-1811.

Sawicki, S.G., Sawicki, D.L., 1986. The effect of loss of regulation of minus-strand RNA synthesis on Sindbis virus replication. Virology 151, 339-349.

Scheidel, L.M., Stollar, V., 1991. Mutations that confer resistance to mycophenolic acid and ribavirin on Sindbis virus map to the nonstructural protein nsP1. Virology 181, 490-499.

Scholte, F.E., Tas, A., Albulescu, I.C., Zusinaite, E., Merits, A., Snijder, E.J., van Hemert, M.J., 2015. Stress granule components G3BP1 and G3BP2 play a proviral role early in Chikungunya virus replication. J Virol 89, 4457-4469.

Scholte, F.E., Tas, A., Martina, B.E., Cordioli, P., Narayanan, K., Makino, S., Snijder, E.J., van Hemert, M.J., 2013. Characterization of synthetic Chikungunya viruses based on the consensus sequence of recent E1-226V isolates. PLoS One 8, e71047. 
Shin, G., Yost, S.A., Miller, M.T., Elrod, E.J., Grakoui, A., Marcotrigiano, J., 2012. Structural and functional insights into alphavirus polyprotein processing and pathogenesis. Proc Natl Acad Sci U S A 109, 16534-16539.

Shirako, Y., Strauss, E.G., Strauss, J.H., 2000. Suppressor mutations that allow sindbis virus RNA polymerase to function with nonaromatic amino acids at the $\mathrm{N}$-terminus: evidence for interaction between nsP1 and nsP4 in minus-strand RNA synthesis. Virology 276, 148-160.

Shirako, Y., Strauss, E.G., Strauss, J.H., 2003. Modification of the $5^{\prime}$ terminus of Sindbis virus genomic RNA allows nsP4 RNA polymerases with nonaromatic amino acids at the $\mathrm{N}$ terminus to function in RNA replication. J Virol 77, 2301-2309.

Shirako, Y., Strauss, J.H., 1994. Regulation of Sindbis virus RNA replication: uncleaved P123 and nsP4 function in minus-strand RNA synthesis, whereas cleaved products from P123 are required for efficient plus-strand RNA synthesis. J Virol 68, 1874-1885.

Shirako, Y., Strauss, J.H., 1998. Requirement for an aromatic amino acid or histidine at the $\mathrm{N}$ terminus of Sindbis virus RNA polymerase. J Virol 72, 2310-2315.

Siegel, R.W., Adkins, S., Kao, C.C., 1997. Sequence-specific recognition of a subgenomic RNA promoter by a viral RNA polymerase. Proc Natl Acad Sci U S A 94, 11238-11243.

Smee, D.F., Alaghamandan, H.A., Kini, G.D., Robins, R.K., 1988. Antiviral activity and mode of action of ribavirin 5'-sulfamate against Semliki Forest virus. Antiviral Res 10, 253-262.

Spuul, P., Balistreri, G., Hellström, K., Golubtsov, A.V., Jokitalo, E., Ahola, T., 2011. Assembly of alphavirus replication complexes from RNA and protein components in a novel trans-replication system in mammalian cells. J Virol 85, 4739-4751.

Spuul, P., Balistreri, G., Kääriäinen, L., Ahola, T., 2010. Phosphatidylinositol 3-kinase-, actin-, and microtubule-dependent transport of Semliki Forest Virus replication complexes from the plasma membrane to modified lysosomes. J Virol 84, 7543-7557.

Spuul, P., Salonen, A., Merits, A., Jokitalo, E., Kääriäinen, L., Ahola, T., 2007. Role of the amphipathic peptide of Semliki forest virus replicase protein nsP1 in membrane association and virus replication. J Virol 81, 872-883.

Sreejith, R., Rana, J., Dudha, N., Kumar, K., Gabrani, R., Sharma, S.K., Gupta, A., Vrati, S., Chaudhary, V.K., Gupta, S., 2012. Mapping interactions of Chikungunya virus nonstructural proteins. Virus Res 169, 231-236.

Sreevalsan, T., Yin, F.H., 1969. Sindbis virus-induced viral ribonucleic acid polymerase. J Virol 3, 599604.

Stapleford, K.A., Miller, D.J., 2010. Role of cellular lipids in positive-sense RNA virus replication complex assembly and function. Viruses 2, 1055-1068.

Stapleford, K.A., Rozen-Gagnon, K., Das, P.K., Saul, S., Poirier, E.Z., Blanc, H., Vidalain, P.O., Merits, A., Vignuzzi, M., 2015. Viral Polymerase-Helicase Complexes Regulate Replication Fidelity To Overcome Intracellular Nucleotide Depletion. J Virol 89, 11233-11244.

Strauss, J.H., Strauss, E.G., 1994. The alphaviruses: gene expression, replication, and evolution. Microbiological reviews 58, 491-562.

Strauss, J.H., Strauss, E.G., 1997. Recombination in Alphaviruses. Seminars in VIROLOGY 8, 85-94. Suopanki, J., Sawicki, D.L., Sawicki, S.G., Kääriäinen, L., 1998. Regulation of alphavirus 26S mRNA transcription by replicase component nsP2. J Gen Virol 79 ( Pt 2), 309-319.

Takkinen, K., 1986. Complete nucleotide sequence of the nonstructural protein genes of Semliki Forest virus. Nucleic Acids Res 14, 5667-5682.

Thaa, B., Biasiotto, R., Eng, K., Neuvonen, M., Gotte, B., Rheinemann, L., Mutso, M., Utt, A., Varghese, F., Balistreri, G., Merits, A., Ahola, T., McInerney, G.M., 2015. Differential Phosphatidylinositol-3-Kinase-Akt-mTOR Activation by Semliki Forest and Chikungunya Viruses Is Dependent on nsP3 and Connected to Replication Complex Internalization. J Virol 89, 11420-11437. Thal, M.A., Wasik, B.R., Posto, J., Hardy, R.W., 2007. Template requirements for recognition and copying by Sindbis virus RNA-dependent RNA polymerase. Virology 358, 221-232. 
Tomar, S., Hardy, R.W., Smith, J.L., Kuhn, R.J., 2006. Catalytic core of alphavirus nonstructural protein nsP4 possesses terminal adenylyltransferase activity. J Virol 80, 9962-9969. Utt, A., Quirin, T., Saul, S., Hellström, K., Ahola, T., Merits, A., 2016. Versatile Trans-Replication Systems for Chikungunya Virus Allow Functional Analysis and Tagging of Every Replicase Protein. PLoS One 11, e0151616. van der Heijden, M.W., Bol, J.F., 2002. Composition of alphavirus-like replication complexes: involvement of virus and host encoded proteins. Arch Virol 147, 875-898. Wang, Y.F., Sawicki, S.G., Sawicki, D.L., 1994. Alphavirus nsP3 functions to form replication complexes transcribing negative-strand RNA. J Virol 68, 6466-6475. Varshavsky, A., 1992. The N-end rule. Cell 69, 725-735. Vasiljeva, L., Merits, A., Golubtsov, A., Sizemskaja, V., Kääriäinen, L., Ahola, T., 2003. Regulation of the sequential processing of Semliki Forest virus replicase polyprotein. J Biol Chem 278, 4163641645.

Weaver, S.C., Lecuit, M., 2015. Chikungunya virus and the global spread of a mosquito-borne disease. N Engl J Med 372, 1231-1239.

Weiss, B.G., Schlesinger, S., 1991. Recombination between Sindbis virus RNAs. J Virol 65, 4017-4025. Weston, J., Villoing, S., Bremont, M., Castric, J., Pfeffer, M., Jewhurst, V., McLoughlin, M., Rodseth, O., Christie, K.E., Koumans, J., Todd, D., 2002. Comparison of two aquatic alphaviruses, salmon pancreas disease virus and sleeping disease virus, by using genome sequence analysis, monoclonal reactivity, and cross-infection. J Virol 76, 6155-6163. 


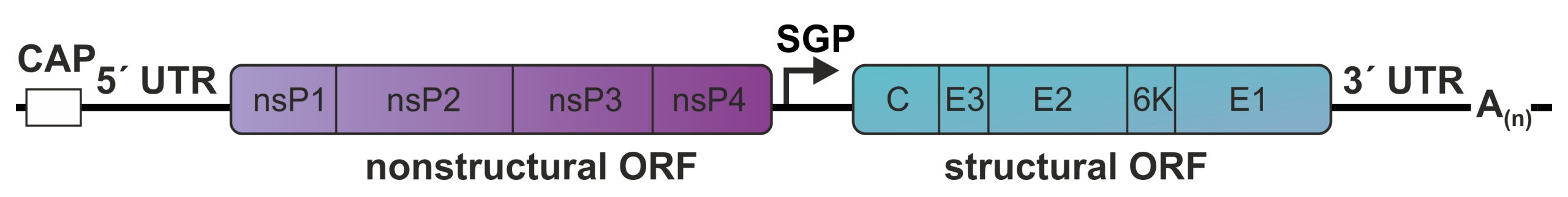




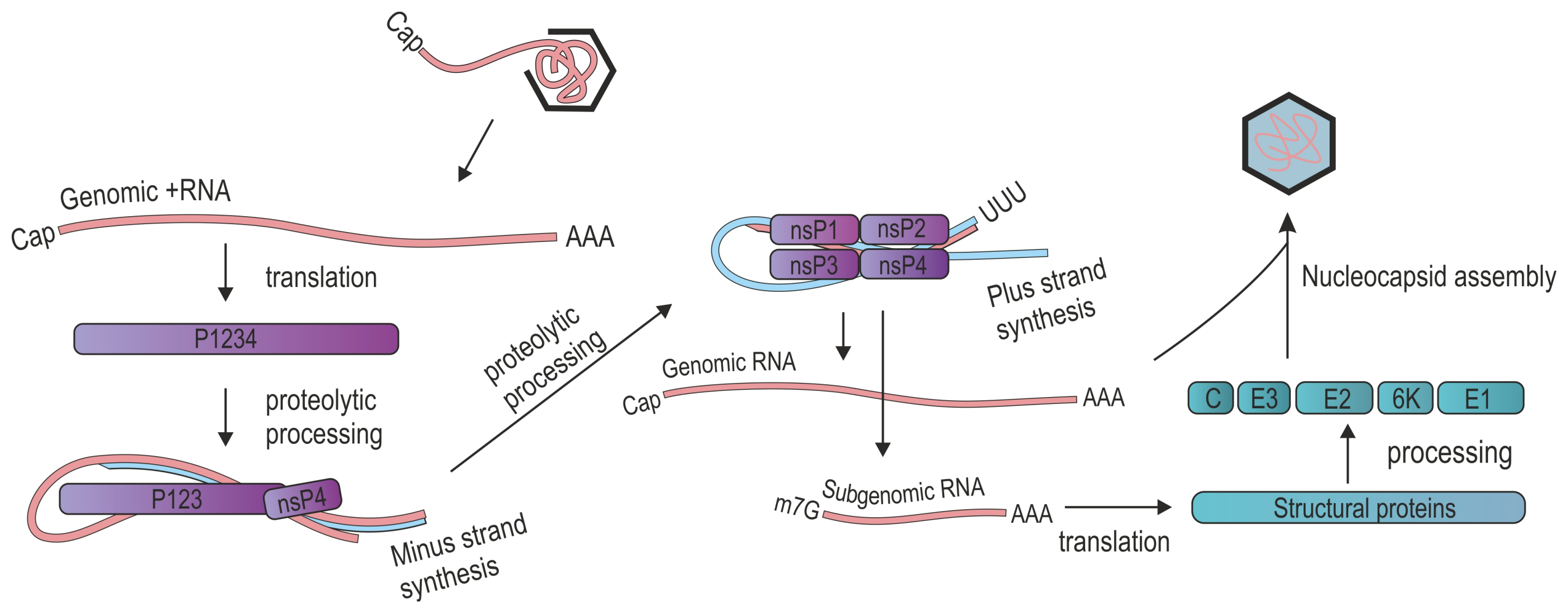




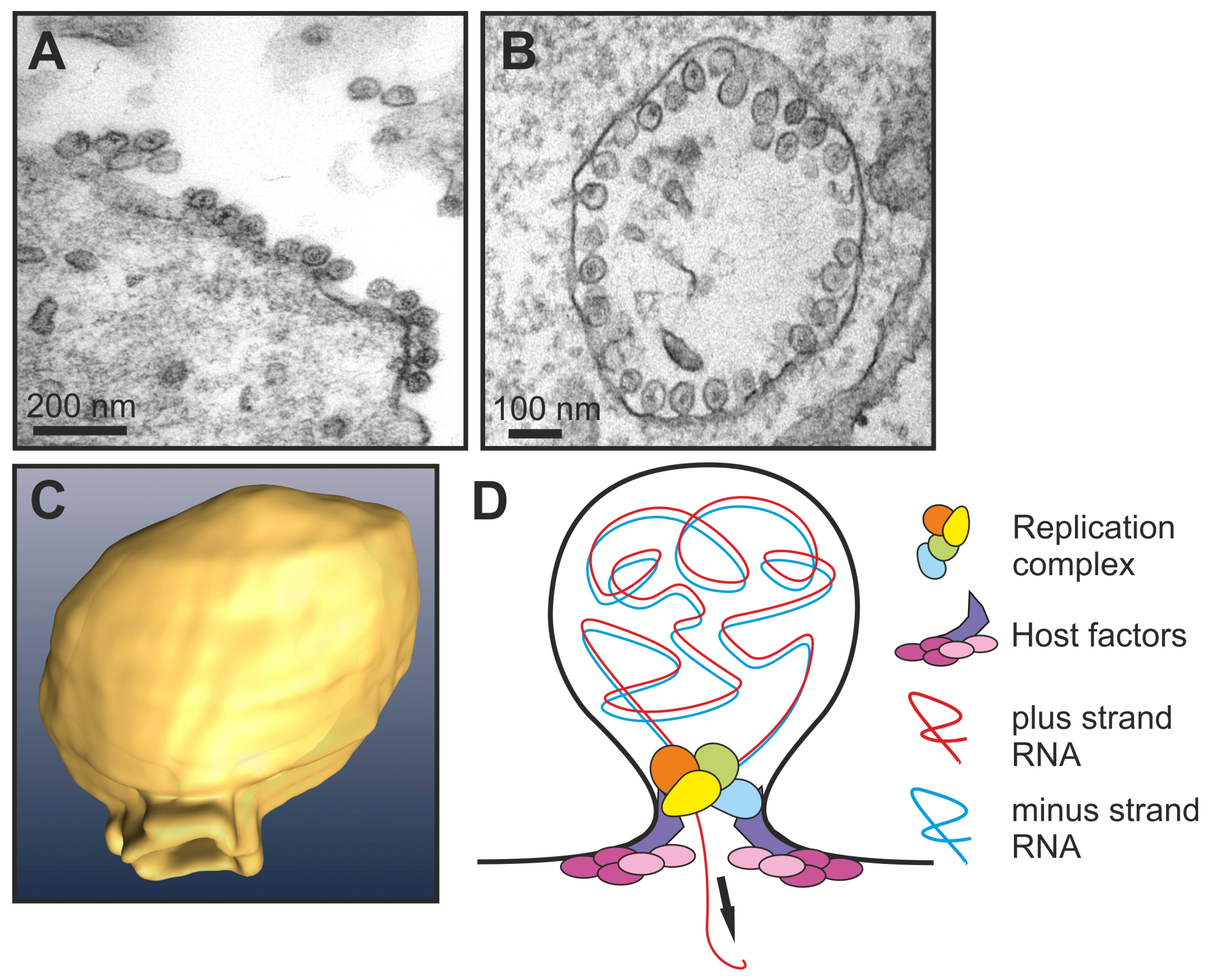




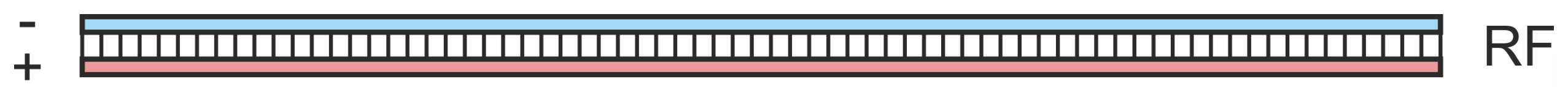

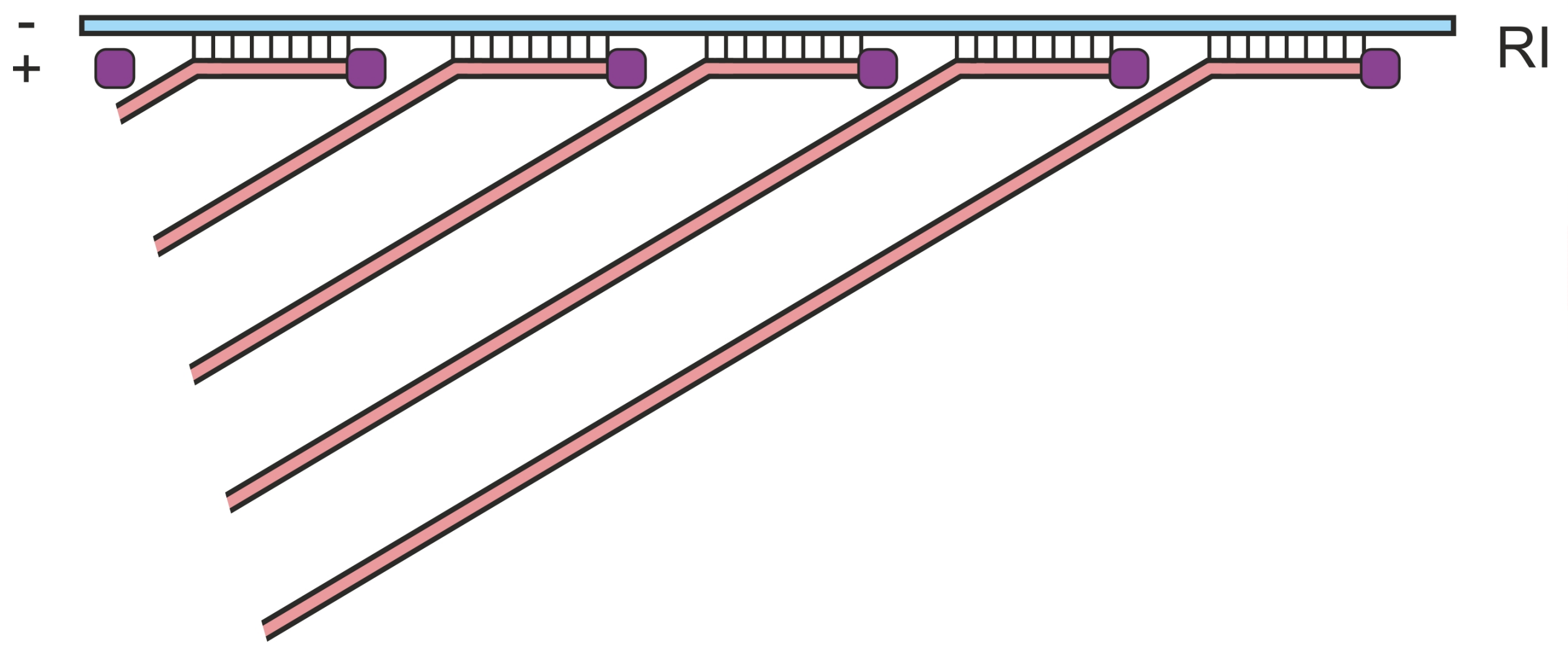




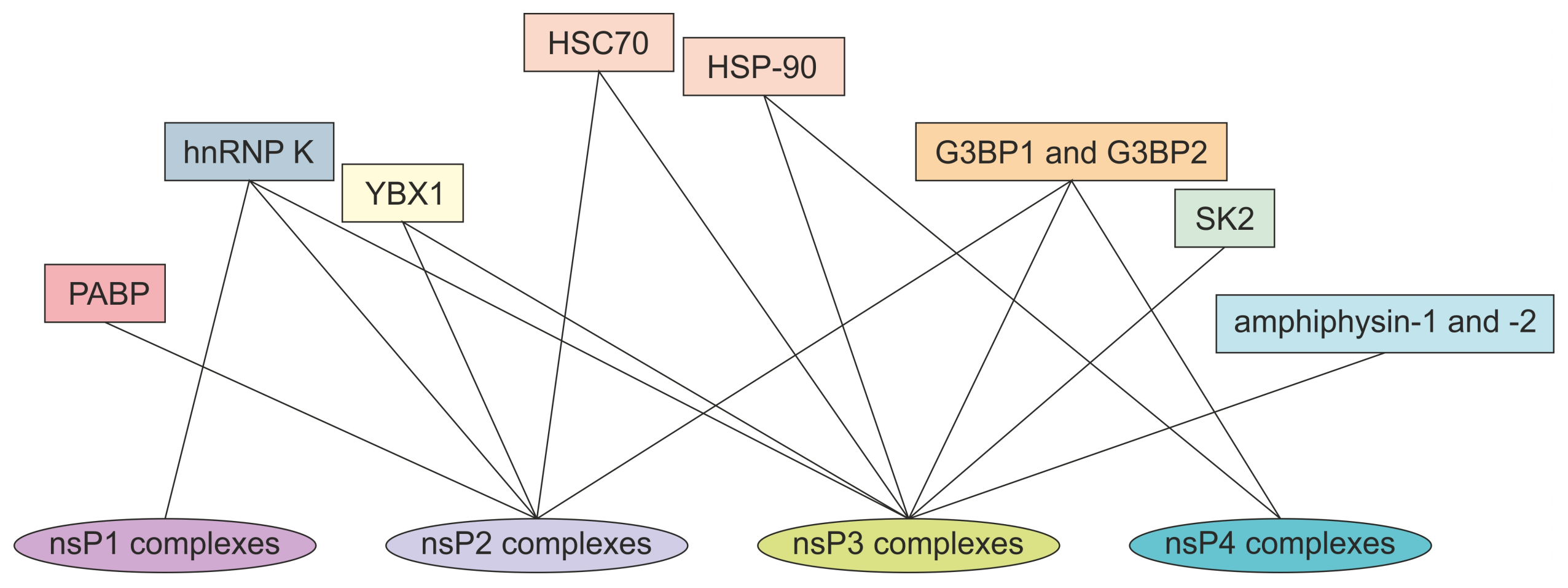


- Alphavirus core polymerase subunit nsP4 is unable to synthesize RNA on its own.

- Processing of the replicase polyprotein regulates the stages of RNA replication.

- RNA replication takes place in special membrane invaginations known as spherules.

- High-resolution structures for polymerase and the membranous complex are lacking. 